\title{
Transgenic systems for unequivocal identification of cardiac myocyte nuclei and analysis of cardiomyocyte cell cycle status
}

\author{
Alexandra Raulf ${ }^{1} \cdot$ Hannes Horder $^{1} \cdot$ Laura Tarnawski $^{2,3} \cdot$ Caroline Geisen $^{1}$ • \\ Annika Ottersbach $^{1} \cdot$ Wilhelm Röll $^{4} \cdot$ Stefan Jovinge $^{2,3} \cdot$ Bernd K. Fleischmannn $^{1,5}$. \\ Michael Hesse ${ }^{1}$
}

Received: 19 December 2014/Revised: 20 March 2015/Accepted: 17 April 2015/Published online: 30 April 2015

(C) The Author(s) 2015. This article is published with open access at Springerlink.com

\begin{abstract}
Even though the mammalian heart has been investigated for many years, there are still uncertainties in the fields of cardiac cell biology and regeneration with regard to exact fractions of cardiomyocytes (CMs) at different developmental stages, their plasticity after cardiac lesion and also their basal turnover rate. A main shortcoming is the accurate identification of $\mathrm{CM}$ and the demonstration of $\mathrm{CM}$ division. Therefore, an in vivo model taking advantage of a live reporter-based identification of $\mathrm{CM}$ nuclei and their cell cycle status is needed. In this technical report, we describe the generation and characterization of embryonic stem cells and transgenic mice expressing a fusion protein of human histone $2 \mathrm{~B}$ and the red fluorescence protein mCherry under control of the CM specific $\alpha \mathrm{MHC}$ promoter. This fluorescence label allows unequivocal identification and quantitation of CM nuclei and nuclearity in isolated cells and native tissue slices. In ventricles of adults, we determined a
\end{abstract}

Electronic supplementary material The online version of this article (doi:10.1007/s00395-015-0489-2) contains supplementary material, which is available to authorized users.

Bernd K. Fleischmann

Bernd.Fleischmann@uni-bonn.de

Michael Hesse

mhesse1@uni-bonn.de

1 Institute of Physiology I, Life and Brain Center, University of Bonn, Sigmund-Freud-Strasse 25, 53105 Bonn, Germany

2 Lund Strategic Research Center for Stem Cell Biology and Cell Therapy, Lund University, Lund, Sweden

3 DeVos Cardiovascular Research Program, Van Andel Institute/Spectrum Health, Grand Rapids, USA

4 Department of Cardiac Surgery, University of Bonn, Bonn, Germany

5 Pharma Center Bonn, Bonn, Germany fraction of $<20 \%$ CMs and binucleation of 77-90\%, while in atria a $\mathrm{CM}$ fraction of $30 \%$ and a binucleation index of $14 \%$ were found. We combined this transgenic system with the CAG-eGFP-anillin transgene, which identifies cell division and established a novel screening assay for cell cycle-modifying substances in isolated, postnatal CMs. Our transgenic live reporter-based system enables reliable identification of $\mathrm{CM}$ nuclei and determination of $\mathrm{CM}$ fractions and nuclearity in heart tissue. In combination with CAG-eGFP-anillin-mice, the cell cycle status of CMs can be monitored in detail enabling screening for proliferationinducing substances in vitro and in vivo.

Keywords Cardiac myocyte $\cdot$ Cell cycle $\cdot$ Genetically altered mice $\cdot$ Proliferation $\cdot$ miRNA

\section{Introduction}

The mammalian heart is composed of different cell types such as endothelial cells, fibroblasts, smooth muscle cells, and cardiomyocytes (CMs). The latter provide the contractile properties of the heart muscle and CM loss in pathologies, such as myocardial infarction or cardiomyopathy, leads to functional impairment and eventually heart failure [16]. While there is mutual consent that CMs make up the majority of the heart mass [20], there is a discrepancy regarding the fraction of $\mathrm{CMs}$ in the mammalian heart which is estimated to be composed of $15-35 \%$ CMs [25, $37,42]$, as addressed by histology but not specific cell markers [3]. A recent report, using flow cytometry, claims that mouse hearts exceptionally consist of $55 \%$ of CMs [2]. Also identifying CM nuclei is challenging in cardiac sections in which CM cytoplasm is identified either by immunohistological staining for structural proteins or by 
transgenic CM-specific overexpression of fluorescence proteins. In those sections, non-CM nuclei in close proximity to $\mathrm{CM}$ cytoplasm cannot be distinguished from $\mathrm{CM}$ nuclei leading to false positives [1].

The majority of CMs are polyploid, caused either by an increase in nuclearity with $\sim 90 \%$ binuclear ventricular CMs in mouse [37, 43] and rat [21], and 25.5-61.9\% in humans $[8,29]$ or by increased nuclear ploidy up to $8 \mathrm{~N}$ in humans [24] and $16 \mathrm{~N}$ in mice [43]. Polyploid cells are thought to be unable to undergo cell division, but are still able to enter the cell cycle and to perform DNA synthesis resulting in CMs with further increased ploidy [14, 23]. Heart regeneration is commonly analyzed with proliferation markers localized in the nucleus, such as PCNA, Ki-67, or pHH3 or Thymidine analogs such as BrdU or ${ }^{3} \mathrm{H}-\mathrm{Thymi}$ dine. The results of these studies depend strongly on the unequivocal identification of CM nuclei, which is errorprone without the use of a specific nuclear CM marker [1]. This could be one of the major reasons for the discrepancies concerning the estimates of annual turnover rates of adult mouse CMs, ranging from $0.76 \%$ [33] up to $80 \%$ [17] and the regeneration potential after experimental lesions, estimated from $0 \%[15,44]$ up to $15.3 \%$ [18]. Using a new in vivo proliferation marker, the eGFP-anillin system, we recently showed that adult $\mathrm{CMs}$ undergo endoreduplication (DNA replication without karyokinesis or cytokinesis) rather than cell division after cardiac lesion in mice [15].

Two challenges arise from the above-mentioned discrepancies, firstly the unambiguous identification of $\mathrm{CM}$ nuclei and secondly the confirmation of CM division and not mere cell cycle activity.

Here, we report the generation of a reporter system that directly visualizes $\mathrm{CM}$ nuclei in vitro and in vivo, by fusing the red fluorescence protein mCherry $(\mathrm{mCh})$ to human histone 2B (H2B) and expressing it in CMs by use of the $\alpha$ MHC (Myh6) promoter. We demonstrate that this fusion protein accurately labels CM nuclei and allows the determination of $\mathrm{CM}$ portions and nuclearity during postnatal development of the mouse heart and after cardiac injury. We further combine aMHC-H2B-mCh mice with the CAG-eGFP-anillin mouse line for unambiguous visualization of cell cycle progression and division of postnatal CMs and for establishing a screening assay for cell cyclemodifying substances.

\section{Methods}

\section{Generation of the $\alpha$ MHC-hH2BmCh-IRES-Puro ${ }^{\mathrm{R}}$ vector}

The pIRES2-eGFP vector (CLONTECH Laboratories, Inc.) was digested with AseI and NheI, blunt-ended and religated. Thereby the CMV IE promoter was excised. EGFP was replaced by the Puromycin ${ }^{\mathrm{R}}$ (PuroR) cassette (HindIII-ClaI fragment) of the Cre-Pac vector. The resulting vector (pIRES2-Puro ${ }^{\mathrm{R}}$ ) was opened with BgIII, blunt-ended and subsequently digested with XhoI. The mouse $\alpha \mathrm{MHC}$ promoter [5.5 kb BamHI(blunt)-SalI fragment] of the $\alpha$ MHC-pBK plasmid (kindly provided by J. Robbins, University of Cincinnati, Cincinnati, $\mathrm{OH}$ ) was inserted into the opened pIRES2-Puro ${ }^{\mathrm{R}}$-vector (paMHCIRES2-Puro ${ }^{\mathrm{R}}$ ).

The pCAG-hH2B-mCh-IRES-Puro ${ }^{\mathrm{R}}$ expression vector was a gift from Heiko Lickert, Helmholtz Zentrum München. The vector was digested with NotI, blunt-ended, and cut with AccIII. The excised H2BmCh-IRES-Puro fragment was ligated into the EcoICRI-AccIII opened paMHC-IRES-Puro ${ }^{\mathrm{R}}$ vector, thereby replacing the IRES2sequence and part of the Puro ${ }^{\mathrm{R}}$ cDNA of the vector. The resulting vector $\alpha \mathrm{MHC}-\mathrm{hH} 2 \mathrm{~B}-\mathrm{mCh}-\mathrm{IRES}-\mathrm{Puro}^{\mathrm{R}}$ ( $\alpha \mathrm{MHC}$ $\mathrm{H} 2 \mathrm{~B}-\mathrm{mCh}$ ) was verified by restriction analysis and sequencing.

\section{Generation and cultivation of transgenic ESC clones}

G4 hybrid ESCs [12] were cultured in Knockout-Dulbecco's modified Eagle's medium (DMEM), high-glucose, supplemented with $15 \% \mathrm{v} / \mathrm{v}$ fetal calf serum (FCS), $0.1 \mathrm{mM}$ nonessential amino acids, $2 \mathrm{mg} / \mathrm{ml} \mathrm{L}$-Glutamine, $50 \mu \mathrm{g} / \mathrm{ml}$ each penicillin and streptomycin, $0.1 \mathrm{mM} \beta$ mercaptoethanol, and $500 \mathrm{U} / \mathrm{ml}$ Leucemia inhibitory factor (LIF). The ESCs were kept on irradiated neomycin-resistant mouse embryo fibroblasts (Millipore).

For generation of transgenic ESCs $5 \times 10^{6}$ cells were mixed with $30 \mu \mathrm{g}$ of linearized plasmid DNA in PBS and electroporated at $250 \mathrm{~V}$ and $500 \mu \mathrm{F}, 1$ pulse, using a BioRad Gene Pulser. The cells were plated on two 100-mm plates. Selection for neomycin-resistant cells started 2 days after electroporation by adding $165 \mu \mathrm{g} / \mathrm{ml} \mathrm{G418}$ to the medium. Resistant colonies were picked onto mouse embryonic fibroblast-coated 24 -well plates, propagated and analyzed for $\mathrm{mCh}$ expression.

\section{Differentiation of transgenic ESCs and purification of CMs}

ESC differentiation was performed using mass culture. In brief, $2.0 \times 10^{6}$ ESCs were suspended in $10 \mathrm{ml}$ differentiation medium (Iscove's Modified Dulbecco's Medium (IMDM), supplemented with $20 \% \quad$ FCS, $0.1 \mathrm{mM}$ nonessential amino acids, $50 \mu \mathrm{g} / \mathrm{ml}$ each penicillin and streptomycin, $0.1 \mathrm{mM} \beta$-mercaptoethanol in the absence of LIF and cultivated on a horizontal shaker at $70-80 \mathrm{rpm}$, $37{ }^{\circ} \mathrm{C}$, and $5 \% \mathrm{CO}_{2}$ for 2 days. Half of the EBs were further cultivated in a CELLSPIN 500 (INTEGRA biosciences) 
spinner flask. To select for CMs, puromycin treatment $(0.5-1.5 \mu \mathrm{g} / \mathrm{ml}$ depending on the resistance of particular clone) was started on day 10 of differentiation in suspension culture. For isolation of CMs on d11 and d13, the EBs were dissociated with $0.125 \%$ Trypsin/EDTA for $5 \mathrm{~min}$. Treatment was stopped with differentiation medium and the cells were centrifuged $(210 \mathrm{~g}, 5 \mathrm{~min}) . \sim 10^{5}$ cells were plated on coverslips, pre-treated with $10 \mu \mathrm{g} / \mathrm{ml}$ fibronectin in 24 -well tissue plates, cultured for $48 \mathrm{~h}$ in differentiation medium, and for further purification of CMs puromycin was added. Cells were fixed with $4 \%$ paraformaldehyde (PFA) at room temperature (RT) for $30 \mathrm{~min}$.

\section{Copy number determination by qPCR}

The copy number of the $\mathrm{H} 2 \mathrm{~B}-\mathrm{mCh}$ transgene was determined in different ESC clones applying a TaqMan-based qPCR assay as described by the manufacturer (Applied Biosystems). For the transgene detection, a Carboxyfluorescein (FAM)-coupled $\mathrm{mCh}$ probe (Applied Biosytems) was used and as genomic reference a VICcoupled probe for the Transferrin receptor was chosen. Both probes were analyzed in triplicates for each gDNA (200 ng). QPCR was performed in a Rotor-Gene 6000 (Corbett).

\section{Transgenic $\alpha$ MHC-H2BmCh-IRES-Puro ${ }^{\mathrm{R}}$ mice and myocardial infarction}

All animal experiments were performed in accordance with the guidelines from Directive 2010/63/EU of the European Parliament on the protection of animals used for scientific purposes and were approved by the Animal Care Committees (8.87-50.10.31.08.199 and 8.87-50.10.37.09.238). Transgenic ESC clones were screened for a proper karyotype (40 chromosomes) and aggregated with diploid morula stage CD1 embryos as described previously [26]. Ventricular cryolesions were generated in 10-week-old mice with 3.5-mm liquid-nitrogen-cooled copper probes as described earlier [31]. Briefly, mice were anesthetized with $4 \mathrm{Vol} \%$ Isoflurane, $0.6 \mathrm{l} / \mathrm{min} \mathrm{O}_{2}, 0.4 \mathrm{l} / \mathrm{min} \mathrm{N}_{2} \mathrm{O}$, intubated and ventilated. The concentration of Isoflurane was reduced to 1-2 Vol\%. The heart was exposed by thoracotomy. For local anesthesia, $0.1 \mathrm{ml}$ of a $0.5 \%$ Bupivacaine solution was injected into the intercostal musculature. Cryolesions at the anterolateral left ventricular wall were generated using liquid-nitrogen-cooled copper probes $3.5 \mathrm{~mm}$ in diameter. For ligation the left anterior descending (LAD) coronary artery was occluded directly distal of the left atrial auricle. For peri-operative analgesia, $20 \mathrm{mg} / \mathrm{kg} \mathrm{s.c} \mathrm{Metamizol} \mathrm{was} \mathrm{used} \mathrm{and} 5 \mathrm{mg} / \mathrm{kg}$ s.c. Carprofen was used for analgesia post operation for 5 days.
Neonatal mice were euthanized by decapitation and adult mice by cervical dislocation.

\section{Left ventricular catheterization and hemodynamics}

Left ventricular catheterization was performed on 15-week-old H2B-mCh and CD1 wt mice by a blinded investigator (for detail see [11]). Briefly, a 1.4 F Millar Aria1 catheter (Millar Instruments Inc., Houston, USA) was inserted under general anesthesia via the right carotid artery into the left ventricle, and pressure-volume (PV)loops were recorded online. For analysis, Millar PVAN software was used. After the experiment, mice were sacrificed and hearts were harvested for histological analysis.

\section{Dissociation of postnatal mouse hearts and miRNA transfection}

Mice were sacrificed on $\mathrm{d} 2 / \mathrm{d} 3 / \mathrm{d} 7$ after birth; the hearts were prepared and analyzed for $\mathrm{H} 2 \mathrm{~B}-\mathrm{mCh}$ expression by using a macroscope (AxioZoom.V16, Zeiss). Atria and ventricles of the transgenic hearts were separated and dissociated using the Neonatal heart dissociation kit (Miltenyi Biotec) without using the gentle MACS Dissociator. The heart tissue was dissected by slowly pipetting every $15 \mathrm{~min} .7500$ cells were seeded per well on a $0.001 \%$ fibronectin-coated 384-well $\mu$-clear microtiter plate (Greiner). Cells were incubated in differentiation medium (see above) at $37{ }^{\circ} \mathrm{C}$ and $5 \% \mathrm{CO}_{2}$. For analysis of the binucleation index, cells were fixated after overnight adhesion to the dish.

In order to transfect postnatal CMs, ventricles of $\alpha \mathrm{MHC}$ H2B-mCh/CAG-eGFP-anillin double transgenic hearts were dissociated as described above. $5 \mu$ l of $500 \mathrm{nM}$ Stock solutions of hsa-miR-199a-3p or miR mimic, Negative control \#1 (Ambion, Life Technologies) were incubated with $0.2 \mu \mathrm{l}$ Lipofectamine RNAi Max (Invitrogen) in $14.8 \mu \mathrm{l}$ OPTI-MEM on $0.001 \%$ fibronectin-coated 384-well $\mu$-clear microtiter plates (Greiner) for $20 \mathrm{~min}$ at RT. Afterward, 7500 dissociated cells were added in a volume of $60 \mu$ differentiation medium. Medium was changed after $48 \mathrm{~h}$ and the cells were further incubated for $24 \mathrm{~h}$.

\section{Harvesting of mouse hearts}

Heparinized adult mice were sacrificed by cervical dislocation and the hearts were harvested. After cannulation of the ascending aorta, the hearts were perfused with PBS, followed by perfusion with $4 \%$ PFA, and further fixation overnight. Embryonic and postnatal hearts were excised and immersion fixated in $4 \%$ PFA. Hearts were dehydrated in $20 \%$ sucrose solution and mounted in O.C.T. compound. 


\section{Langendorff dissociation}

Ventricular myocytes from adult mice were enzymatically isolated by Langendorff perfusion as previously described [39], with minor variations. Briefly, animals were killed by cervical dislocation. The heart was dissected and perfused using oxygenated $\mathrm{Ca}^{2+}$-free Tyrode solution $(135 \mathrm{mM}$ $\mathrm{NaCl}, 4 \mathrm{mM} \mathrm{KCl}, 1 \mathrm{mM} \mathrm{MgCl}$, $2.5 \mathrm{mM}$ HEPES, $5 \mathrm{mM}$ Glucose, $\mathrm{pH}$ 7.4) with $25 \mathrm{mM}$ 2,3-Butanedione monoxime (BDM) for $5 \mathrm{~min}$. The enzymatic digestion with $1 \mathrm{mg} / \mathrm{ml}$ (activity $0.199 \mathrm{U} / \mathrm{mg}$ ) Collagenase $\mathrm{B}$ and $0.033 \mathrm{mg} / \mathrm{ml}$ Trypsin was performed in oxygenated Tyrode solution with $25 \mathrm{mM}$ BDM and $50 \mu \mathrm{M} \mathrm{CaCl}$ for $8-12$ min and after manually dissecting the tissue the reaction was stopped in stopping solution (oxygenated Tyrode solution with $25 \mathrm{mM} \mathrm{BDM}, 50 \mu \mathrm{M} \mathrm{CaCl}_{2}$ and $5 \% \mathrm{FCS}$ ). The cell suspension was filtered through a $100-\mu \mathrm{m}$ Cell strainer and centrifuged (700 rpm, $1 \mathrm{~min}$ ). Isolated cells were plated on $8 \mu \mathrm{g} / \mathrm{ml}$ laminin-coated coverslips in 24-well plates in differentiation medium (see above) at $37{ }^{\circ} \mathrm{C}, 5 \% \mathrm{CO}_{2}$ for $5 \mathrm{~h}$ and fixated with $4 \%$ PFA solution afterward.

\section{Histology and immunofluorescence stainings}

Following PFA fixation, all tissues and EBs were incubated in $20 \%$ sucrose in PBS prior to cryopreservation in Tissue Tek O.C.T. compound (Sakura Finetek Europe B.V.). Sectioning of $10 \mu \mathrm{m} / 50 \mu \mathrm{m}$ cryoslices was performed with a cryotome CM 3050 S (Leica).

Fixated cells and tissue slices were stained for the following differentiation and proliferation markers (in $0.2 \%$ Triton X in PBS, supplemented with $5 \%$ donkey serum; $2 \mathrm{~h}$ at RT): $\alpha$-actinin (1:400, Sigma-Aldrich A7811), cTropT (1:50, DSHB Hybridoma Product RV-C2), PCM-1 (1:200, Cell Signaling G2000), and Aurora B kinase (1:400, SigmaAldrich A5102). For the PCM-1 staining a short antigenretrieval with $10 \mathrm{mM}$ sodium citrate, $\mathrm{pH}$ 6.0, was performed in a KOS Microwave (Milestone, program: $4 \min 70^{\circ} \mathrm{C}$, $3 \min 85^{\circ} \mathrm{C}, 4 \min 90{ }^{\circ} \mathrm{C}, 5 \min 93{ }^{\circ} \mathrm{C}$ ), which allowed conservation of the H2B-mCh fluorescence. Primary antibodies were visualized by secondary antibodies conjugated to Cy5 (1:400, Jackson ImmunoResearch) diluted in $1 \mu \mathrm{g} / \mathrm{ml}$ Hoechst 33342 (nuclei staining) at RT for $1 \mathrm{~h}$. Fluorescein Griffonia Simplificiosa Lectine (GSL, Vector Laboratories) was diluted 1:100 and stained at RT for $1 \mathrm{~h}$. Immunostainings were documented with an inverted fluorescence microscope (Axiovert 200; Carl Zeiss MicroImaging, Inc.) equipped with a slider module (ApoTome; Carl Zeiss MicroImaging, Inc.). Sirius red, hematoxylin, and eosin stainings were performed using standard histology protocols. Pictures were acquired with an AxioStar Plus microscope (Zeiss).

For quantification of $\mathrm{CM}$ nuclei fractions in different regions of the heart, $10-\mu \mathrm{m}$ thick transversal cryo-sections were analyzed (zone 1: section at atria level, zone 2: section through the middle of the ventricle, determined by length measurement of a macroscopic picture of each heart, zone 3: section in $\sim 200 \mu \mathrm{m}$ distance from the apex). The following $25 \times$ pictures of cross-sectioned CMs were analyzed per mouse ( $n=3$ per time point) and time points (P3, P7, $9 \mathrm{~W})$ : zone 1: left $A$ atrium, $L V$ left ventricle compact zone, LV trabecular zone, $R V$ right ventricle compact zone, RV trabecular zone, zone 2: LV compact zone, $\mathrm{LV}$ trabecular zone, $\mathrm{RV}$ compact zone, $\mathrm{RV}$ trabecular zone, layer 3: LV compact zone, LV trabecular zone.

\section{Determination of binuclearity and fractions of $\mathrm{CM}$ nuclei in thick slices}

PFA-fixated, cryopreserved hearts were sliced into $50-\mu \mathrm{m}$ thick sections with a cryotome CM 3050S (Leica) and treated with RNAse A $(20 \mu \mathrm{g} / \mathrm{ml})$ in wash buffer $(0.5 \mathrm{M}$ $\mathrm{NaCl}, 0.1 \mathrm{M}$ Tris $\mathrm{pH} 7.5,50 \mathrm{mM}$ EDTA) for $1 \mathrm{~h}$ at $37{ }^{\circ} \mathrm{C}$. Slices were incubated with $1 \mu \mathrm{M}$ TO-PRO3 iodide (642/ 661) (Molecular Probes) and Fluorescein wheat germ agglutinine (WGA) (1:100, Vector Laboratories) at $4{ }^{\circ} \mathrm{C}$ overnight. Image stacks were acquired with an inverted confocal laser scanning microscope (Nikon Eclipse Ti) equipped with a $\times 40 / 1.15 \mathrm{NA}$ water-dipping objective (ApoLWD 40× WI SDIC N2). Stacks with a z-step width of $0.5 \mu \mathrm{m}$ were recorded. Excitation wavelengths for WGA, H2B-mCh, and TO-PRO 3 were 488, 543, and $642 \mathrm{~nm}$, respectively. Binucleation was determined in z-stacks manually by scrolling through the different layers of the stack. Only cells that lay completely within the z-stack (WGA staining visible in every dimension) were quantified.

The number of $\mathrm{CM}$ nuclei $\left(\mathrm{H} 2 \mathrm{~B}-\mathrm{mCh}^{+}\right)$and all nuclei $\left(\mathrm{TO}^{\mathrm{PRO}} 3^{+}\right.$) was determined in 3D-reconstructions using the automatic 3D analysis module of NIS Elements. The result was verified by manually correcting for doublets, which were nuclei in very close proximity to each other, that were not correctly separated by the software. Virtual longitudinal and cross sections $(0.5 \mu \mathrm{m})$ were generated using NIS Elements. Per thick slice $\geq 3$ longitudinal sections with a distance of $10 \mu \mathrm{m}$ to each other were manually analyzed for $\mathrm{H} 2 \mathrm{~B}-\mathrm{mCh}$ signals and TO-PRO3 signals. In virtual cross sections, the distance between the analyzed sections ( $n \geq 4$ per thick slice) was $40 \mu \mathrm{m}$.

\section{Nuclei isolation and flow cytometry}

Nuclei isolation experiments were performed as reported earlier [5]. In brief, frozen adult H2B-mCh hearts were cut into pieces manually and put in ice cold lysis buffer (in mM: 350 Sucrose; $5 \mathrm{CaCl}_{2} ; 5 \mathrm{C}_{4} \mathrm{H}_{6} \mathrm{MgO}_{4}-4 \mathrm{H}_{2} \mathrm{O} ; 2.0$ EDTA; 0.5 EGTA; 10 Tris-HCl (pH 8.0); 1 DTT). The 
heart pieces were homogenized with a T-25 Ultra-Turrax ${ }^{\circledR}$ probe homogenizer (IKA) at $24 \mathrm{k} \mathrm{rpm}$ for $10 \mathrm{~s}$ and then using a glass dounce to further homogenize the tissue and to free the nuclei. The crude lysate was passed through a 100- and 70- $\mu \mathrm{m}$ nylon mesh cell strainer (BD Biosciences), consecutively, and then centrifuged at $700 \mathrm{~g}$ for $10 \mathrm{~min}$ in $\left(4{ }^{\circ} \mathrm{C}\right)$. The nuclei pellet was re-suspended in sucrose buffer containing (in $\mathrm{mM}$ ) the following: 2100 sucrose; 5 $\mathrm{C}_{4} \mathrm{H}_{6} \mathrm{MgO}_{4}-4 \mathrm{H}_{2} \mathrm{O} ; 1$ DTT; 10 Tris- $\mathrm{HCl}$ (pH 8.0). By using gradient centrifugation, the sample was centrifuged at $13,000 \mathrm{~g}$ for $60 \mathrm{~min}$ in $4{ }^{\circ} \mathrm{C}$. The nuclei pellet was resuspended in nuclei storage buffer containing (in $\mathrm{mM}$ ) the following: 440 sucrose; $70 \mathrm{KCl} ; 10 \mathrm{MgCl}_{2} ; 10$ Tris- $\mathrm{HCl}$ (pH 7.2); 1.5 spermine. Nuclei were stained with rabbit anti-mouse PCM-1 (Cell Signaling Tech.) overnight in $4{ }^{\circ} \mathrm{C}$. Primary antibody was visualized with secondary antibody conjugated to FITC (Jackson ImmunoResearch) and DNA was stained with DAPI. Cytometric acquisition was performed on Aria III (BD Biosciences) using the 70- $\mu \mathrm{m}$ nozzle. Positive gates were set according to unstained controls, FMO controls, and single staining controls. Analysis was done using Flow Jo ${ }^{\mathrm{TM}}$ Version V10.

\section{Statistical analysis}

Data are depicted as mean \pm SEM. Statistical significance was determined by Student's unpaired $t$ test or 1way ANOVA with Bonferroni's multiple comparison test. $p<0.05$ was considered statistically significant.

\section{Results}

\section{Characterization of $\alpha \mathrm{MHC}-\mathrm{hH} 2 \mathrm{~B}-\mathrm{mCh}$-IRES- Puro $^{\mathrm{R}}$ transgenic ESC clones}

To establish a system that allows unequivocal identification of CM nuclei in vitro and in vivo, we cloned a fusion cDNA consisting of human histone $2 \mathrm{~B}(\mathrm{H} 2 \mathrm{~B})$ and $\mathrm{mCh}$ behind the cardiac-specific $\alpha$-Myosin-heavy-chain ( $\alpha \mathrm{MHC}$, Myh6) promoter (Fig. 1a). The construct also contained an internal ribosomal entry site (IRES) followed by a Puromycin resistance cassette for the enrichment of CMs. We used a fusion protein with H2B for two main reasons: First, for determination of the cell cycle activity in CMs nuclear markers such as BrdU or Ki-67 are used most commonly, rendering the clear identification of CM nuclei of crucial importance [1]. Second, after dissolving of the nuclear membrane in M-phase, transgenic systems using a nuclear localization signal encounter a translocation of the fluorescence protein to the cytoplasm, which is hard to detect for cell tracking image analysis algorithms [27]. Additionally, H2B fusion proteins have a very long half- life [40], therefore maintaining the nuclear signal during potentially fluctuating activity of the aMHC promoter.

For generation of transgenic mice and in vitro analysis of $\mathrm{H} 2 \mathrm{~B}-\mathrm{mCh}$ expression in $\mathrm{CMs}$, we generated stably transfected mouse embryonic stem cell (mESC) lines and differentiated these into embryonic bodies (EBs). The expression of the reporter construct became visible around day 8 of differentiation (day 8 ). This correlated with the appearance of the first spontaneous beating cell clusters in the EBs and $\mathrm{H} 2 \mathrm{~B}-\mathrm{mCh}$ expression was restricted to these areas (Fig. 1b, Suppl. video 1). Cryoslices of fixated EBs at day 10 revealed an exclusive expression of the $\mathrm{H} 2 \mathrm{~B}-\mathrm{mCh}$ protein in the nuclei of $\alpha$-actinin ${ }^{+}$CMs (Fig. 1c, d). On day 10 puromycin treatment was started for 3 days to enrich for ESC-derived CMs. During puromycin treatment, EBs were dissociated and the cells replated (Fig. 1e), followed by quantification of $\mathrm{H} 2 \mathrm{~B}-\mathrm{mCh}^{+} / \alpha$-actinin ${ }^{+} \mathrm{CMs}$ (Fig. 1f), which revealed a $\sim 16$-fold enrichment of CMs (Fig. 1g). The overlap of $\mathrm{H} 2 \mathrm{~B}-\mathrm{mCh}$ expression and $\alpha$-actinin staining was $96 \pm 1.5 \%$, revealing high specificity of the transgene. In summary, the $\alpha \mathrm{MHC}-\mathrm{H} 2 \mathrm{~B}-\mathrm{mCh}$ system can identify $\mathrm{CM}$ nuclei in vitro and $\mathrm{CMs}$ derived from the transgenic ESCs are viable and can be enriched by puromycin treatment.

\section{H2B-mCh specifically marks CM nuclei in transgenic hearts}

From a characterized $\alpha \mathrm{MHC}-\mathrm{H} 2 \mathrm{~B}-\mathrm{mCh}$ ESC clone with only one transgene integration, we generated a transgenic mouse line by aggregation with diploid embryos from wildtype CD1 mice. The chimeric mice provided germ-line transmission and the transgenic progeny was viable, fertile, and had a normal lifespan.

Macroscopically, $\mathrm{H} 2 \mathrm{~B}-\mathrm{mCh}$ adult hearts displayed $\mathrm{mCh}$ fluorescence in ventricles and atria (Fig. 2a), and higher magnification showed a restriction of the $\mathrm{H} 2 \mathrm{~B}-\mathrm{mCh}$ expression to nuclei (Fig. 2a, right picture). Cryoslices of these hearts revealed that the expression of the fusion protein was specific for all CM nuclei (Fig. 2b), which were identified by $\alpha$-actinin staining (Fig. 2c).

As a definitive proof that $\mathrm{H} 2 \mathrm{~B}-\mathrm{mCh}$ exclusively marks all CMs, we performed Langendorff dissociation of aMHC-H2B-mCh hearts $(n=6)$ to verify the specificity and the penetrance of our reporter system. All CMs with rod-shaped morphology and positive for $\alpha$-actinin expressed $\mathrm{H} 2 \mathrm{~B}-\mathrm{mCh}$ in their nuclei (Fig. 2d; $n=1610$ ) proving complete penetrance, while nuclei from other cells were negative for $\mathrm{H} 2 \mathrm{~B}-\mathrm{mCh}$ (Fig. 2d, arrows). The specificity to heart tissue was further demonstrated by analyzing sections from skeletal muscles such as M. tibialis anterior and diaphragm, which did not display any $\mathrm{H} 2 \mathrm{~B}-\mathrm{mCh}$ expression (Fig. 2e). 


\section{A $-\alpha \mathrm{MHC}$ hH2B-mCh IRES PuroR - polyA -}
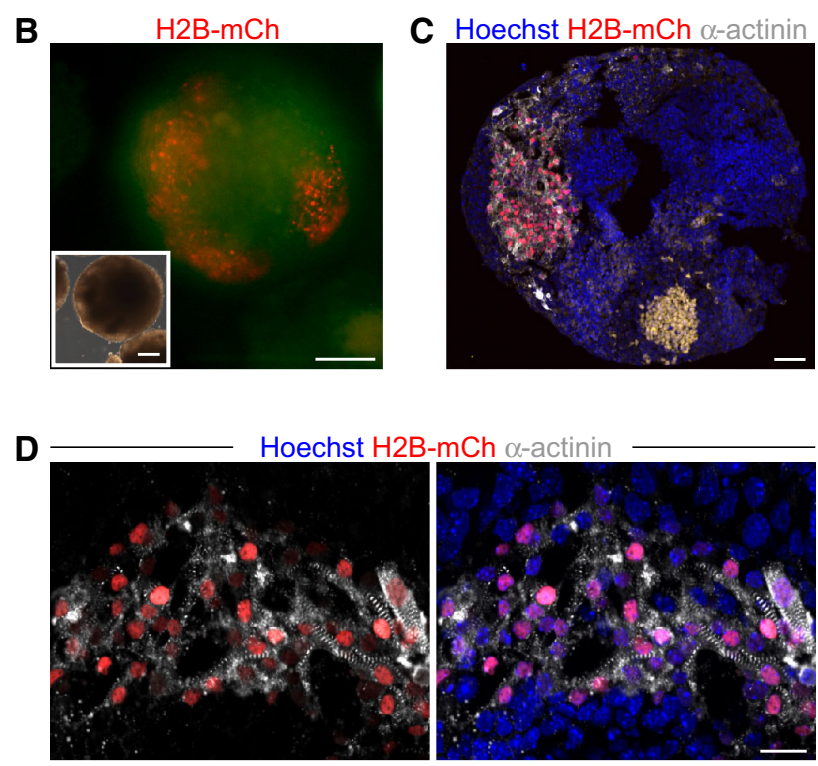

E
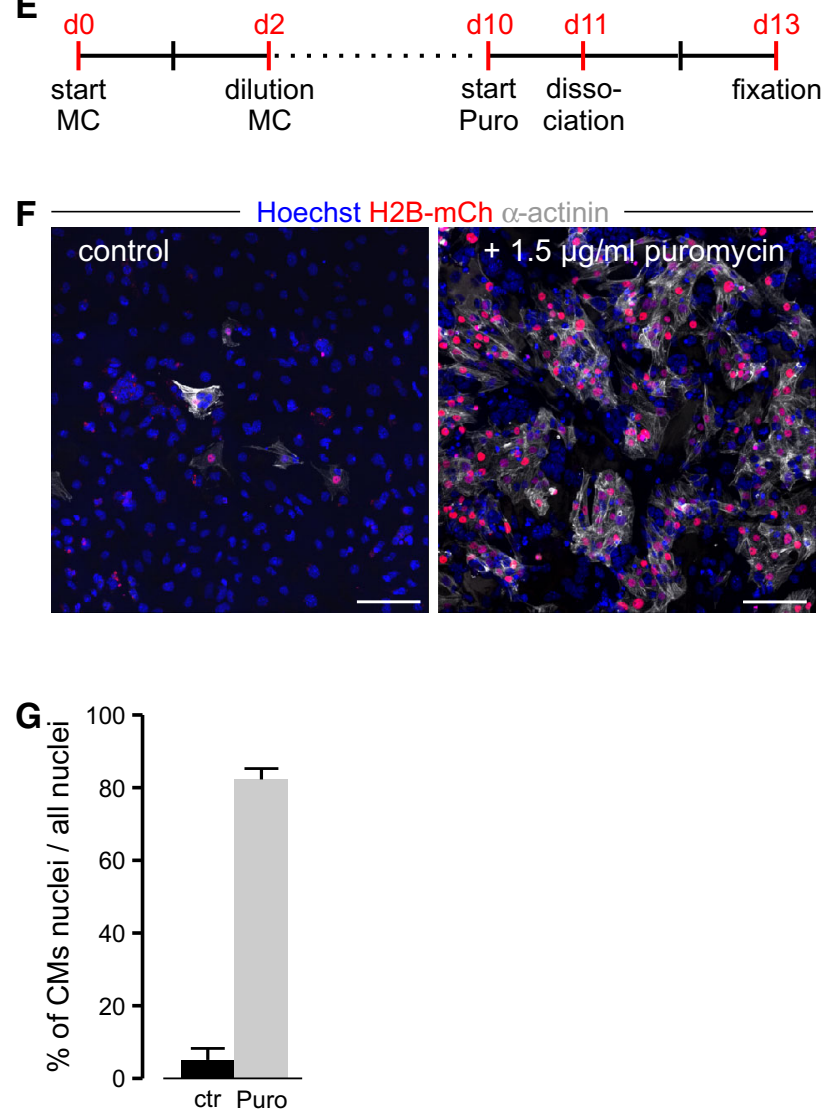

We further investigated the specificity of the H2B-mCh expression pattern by staining with GSL to exclude expression in endothelial cells (Fig. 2f). There were several nuclei, which were covered by cross-striations and
4Fig. 1 Identification of $\mathrm{CM}$ nuclei in vitro. a Expression construct used for the generation of $\alpha \mathrm{MHC}-\mathrm{H} 2 \mathrm{~B}-\mathrm{mCh}$ transgenic mouse ESCs. b $\mathrm{H} 2 \mathrm{~B}-\mathrm{mCh}$ expression in beating areas of $\mathrm{EBs}$ at d14 of differentiation. Scale bar $200 \mu \mathrm{m}$. c Section of an $\mathrm{H} 2 \mathrm{~B}-\mathrm{mCh}^{+} \mathrm{EB}$ indicates specific expression of the fusion protein in CMs, identified by $\alpha$ actinin staining. Scale bar $50 \mu \mathrm{m}$. d Close-up of a CM-rich region of an $\mathrm{H} 2 \mathrm{~B}-\mathrm{mCh}^{+} \mathrm{EB}$. CMs were identified by $\alpha$-actinin staining. Scale bar $20 \mu \mathrm{m}$. e Protocol for differentiation of ESCs and selection for CMs. f Selection for CMs in H2B-mCh transgenic EBs by puromycin treatment. Left picture shows dissociated EBs without selection and right picture with puromycin treatment for 3 days. $\mathrm{H} 2 \mathrm{~B}-\mathrm{mCh}^{+} \mathrm{CMs}$ were verified by $\alpha$-actinin staining. Scale bar $100 \mu \mathrm{m}$. g Quantification of the selection for CMs in $\alpha \mathrm{MHC}-\mathrm{H} 2 \mathrm{~B}-\mathrm{mCh}$ transgenic EBs (d13) treated with puromycin for 3 days $(n=3)$

Fig. 2 Specificity of H2B-mCh expression in CM nuclei of adult transgenic mice. a Macroscopic picture of an $\mathrm{\alpha MHC}-\mathrm{H} 2 \mathrm{~B}-\mathrm{mCh}$ heart at adult stage. Scale bars $500 \mu \mathrm{m}$. Right picture shows a close-up of the ventricular region. Scale bar $100 \mu \mathrm{m}$. b Transversal section of an $\alpha \mathrm{MHC}-\mathrm{H} 2 \mathrm{~B}-\mathrm{mCh}$ heart reveals expression of the fusion protein in nuclei. Scale bar $500 \mu \mathrm{m}$. c Section of an $\alpha$ MHC-H2B-mCh heart stained for $\alpha$-actinin to prove expression of the fusion protein in CMs. Scale bar $20 \mu \mathrm{m}$. d Langendorff dissociation demonstrates expression of H2B-mCh in all CMs. Scale bar $50 \mu \mathrm{m}$. e H2B-mCh is not expressed in nuclei of skeletal muscle cells of H2B-mCh mice. Scale bar $20 \mu \mathrm{m}$. f $\alpha \mathrm{MHC}-\mathrm{H} 2 \mathrm{~B}-\mathrm{mCh}^{+}$heart section stained with GSL to identify endothelial cells. CMs were identified by cross striation in DIC. Arrows mark nuclei that could be misinterpreted as CM nuclei, arrowheads mark cells that would not have been identified as $\mathrm{CM}$ nuclei without the transgene. Scale bar $20 \mu \mathrm{m}$. g Representative flow cytometric analysis of the percentages of $\mathrm{H} 2 \mathrm{~B}-\mathrm{mCh}^{+}$and $\mathrm{PCM}-1^{+}$ single adult $\mathrm{DAPI}^{+}$nuclei. $\mathbf{h}$ Representative flow cytometric analysis of the percentages of $\mathrm{H} 2 \mathrm{~B}-\mathrm{mCh}$ in single adult $\mathrm{DAPI}^{+}$nuclei. i Staining of PCM- 1 and $\alpha$-actinin in a cryo-slice of an $\alpha$ MHC-H2B$\mathrm{mCh}$ heart. The arrow marks a CM nucleus that is not stained by PCM-1. Scale bar $50 \mu \mathrm{m}$

therefore seemingly belonged to $\mathrm{CMs}$, but were clearly H2B-mCh negative (Fig. 2f, arrows) and vice versa (Fig. 2f, arrowheads). This underlines the difficulty to unequivocally identify $\mathrm{CM}$ nuclei on tissue sections.

Recent studies on cardiac turnover relied on the correct identification of $\mathrm{CM}$ nuclei by antibody staining for specific markers such as Troponin I and T [4] and PCM-1 [6], followed by flow cytometry analysis. To test, if our system can be used to identify CM nuclei out of total nuclei from hearts, we isolated cardiac nuclei from adult H2B$\mathrm{mCh}$ mice and analyzed these by flow cytometry.

$\mathrm{H} 2 \mathrm{~B}-\mathrm{mCh}^{+}$nuclei were easily detectable by flow cytometry and contributed to $25.0 \pm 4.2 \%$ of total heart nuclei (Fig. $2 \mathrm{~g}$ ). To assess the accuracy of the aforementioned nuclear $\mathrm{CM}$ markers, we exemplarily stained nuclei from $\mathrm{H} 2 \mathrm{~B}-\mathrm{mCh}^{+}$hearts for PCM-1. Flow cytometric analysis of the stained nuclei revealed a distinct CMpopulation for $\mathrm{H} 2 \mathrm{~B}-\mathrm{mCh}$, while the $\mathrm{PCM}-1$ staining displayed a broad spreading (Fig. 2h); the correlation between the two markers was $90 \%$, (Fig. 2h). Staining for PCM-1 on tissue sections of adult mouse hearts also revealed a good overlap with the H2B-mCh signal (Fig. 2i). However, 
A

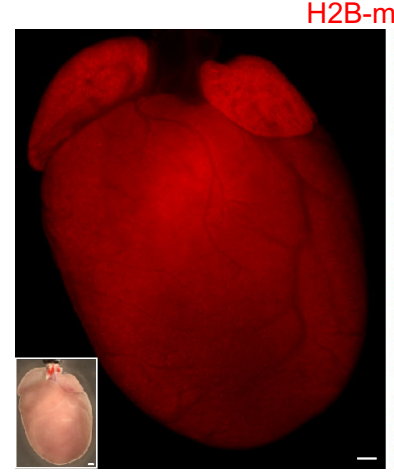

C

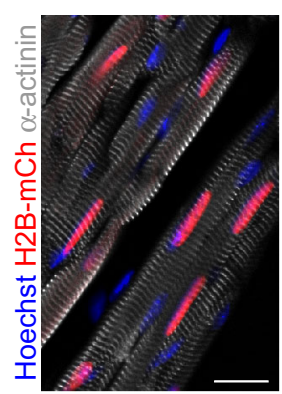

D

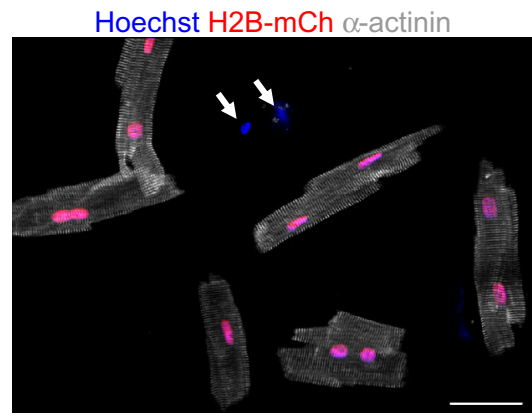

$\mathbf{F}$

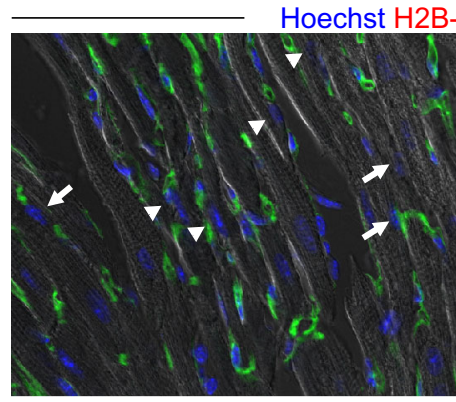

H
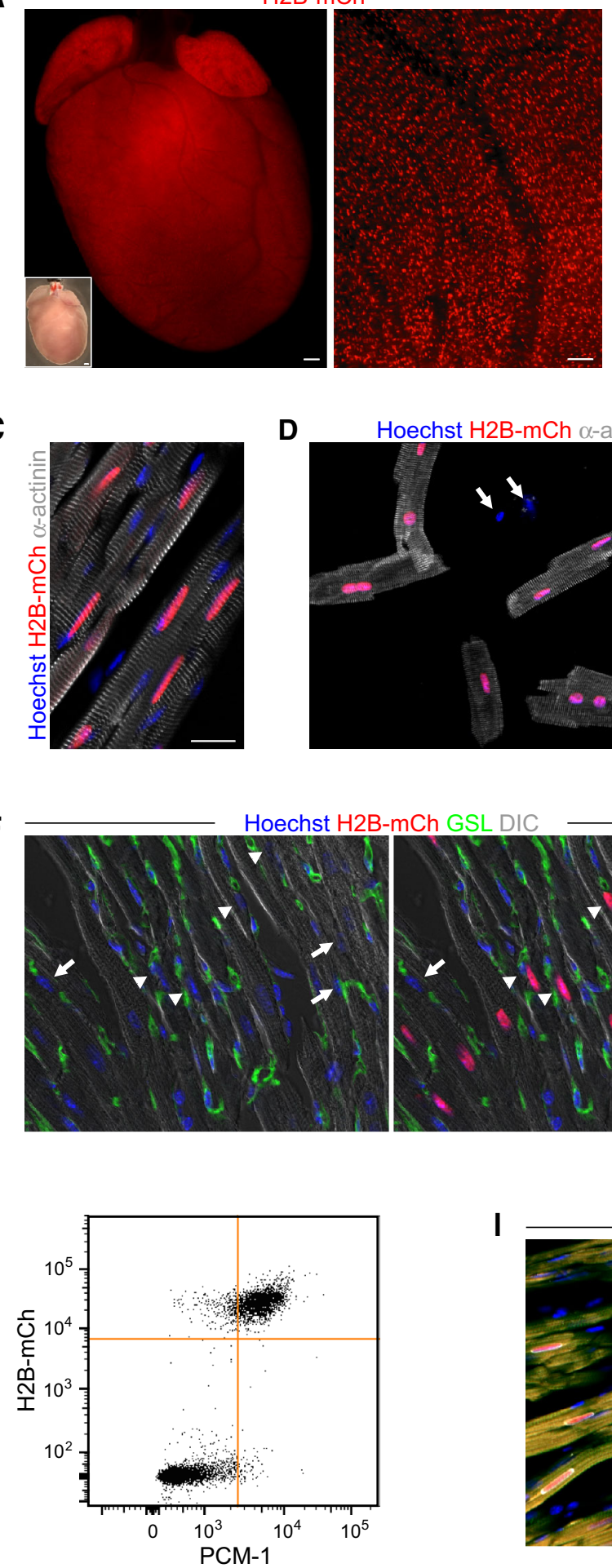

B

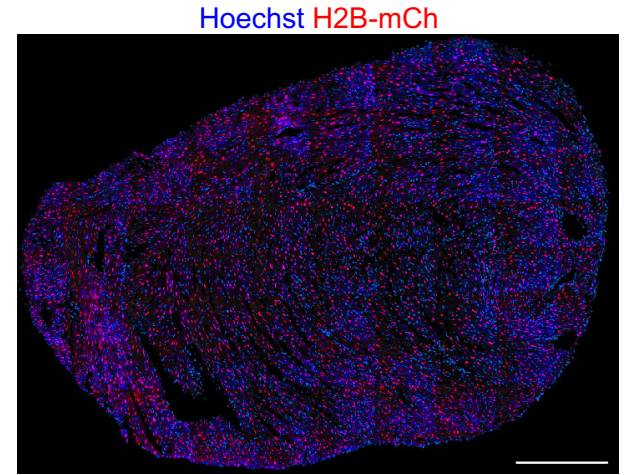

E

Hoechst H2B-mCh autofluo

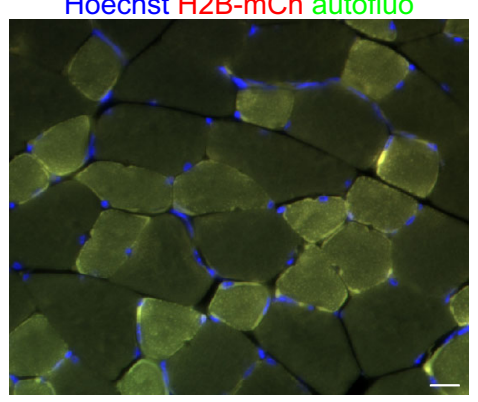

G
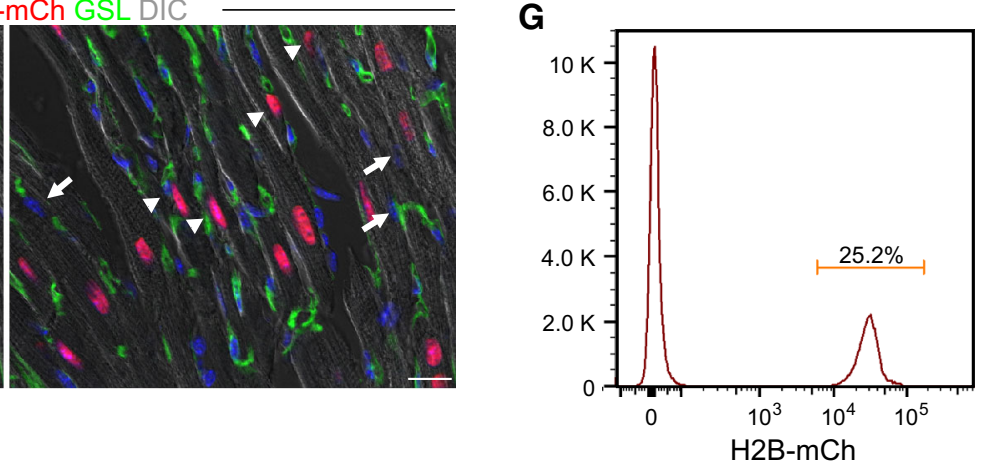

Hoechst $\alpha$-actinin H2B-mCh PCM-1

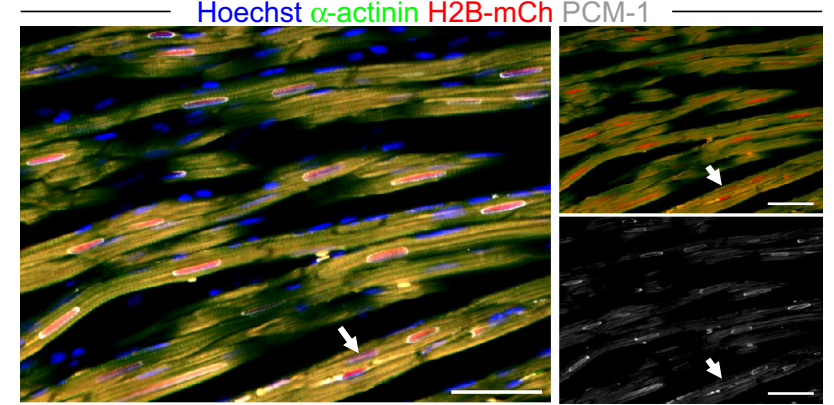

not all of the CM nuclei could be clearly identified by PCM-1 staining (Fig. 2i, arrow). To further exclude possible side effects of the transgene on CMs, we performed morphometric analysis on Langendorff dissociated adult CMs and excluded a hypertrophic effect of transgene expression (Fig. 3a). Also the percentage of binucleated adult CMs in H2B-mCh mice [89.5 $\pm 2 \%(n=4)]$ matched published numbers [21, 43]. Accordingly, 10-weekold transgenic mice did not show any obvious differences in heart weight (Fig. 3b) compared to their wildtype 
littermates. Transgene stability was monitored for 6 generations in mice from a mixed 129S6/SvEvTac $\times$ C57BL/ $6 \mathrm{Ncr} \times \mathrm{CD}-1$ background and for 2 generations of backcrosses into C57BL/6 background. No changes in specificity or intensity of $\mathrm{H} 2 \mathrm{~B}-\mathrm{mCh}$ expression were noted and no detrimental effects on cardiac function and hemodynamics were observed (Table 1); in addition, no fibrosis (Fig. 3c) could be detected.

Next, we analyzed transgene expression during embryonic development and neonatal stages. At E12.5, E15.5, and E18.5, the expression of H2B-mCh was limited mainly to the atria (Fig. 3d), probably due to the restricted activity of the $\alpha \mathrm{MHC}$ promoter [22]. Although there was some expression in the trabecular zone of the left and right ventricles (Fig. 3e), the H2B-mCh signals were only detectable in a subpopulation of $\mathrm{CMs}$ and expression was significantly weaker compared to atrial CMs.

In neonatal mice, transgene expression was found to be already increased in ventricular CMs compared to late embryonic hearts (P0 in Fig. 4a) and on P3 solid expression of the $\mathrm{H} 2 \mathrm{~B}-\mathrm{mCh}$ protein was detectable in ventricular and atrial CMs (P3 in Fig. 4a), as demonstrated by high overlap of $\alpha$-actinin staining and H2B-mCh expression after dissociation (Fig. 4b).

After demonstrating the high specificity and complete penetrance of our transgene for CMs, we applied our system to several open biological questions concerning $\mathrm{CM}$ fractions, ploidy, and cell cycle status.

\section{Determination of CM nuclei fractions at postnatal stages}

One focus of our study was the precise determination of the $\mathrm{CM}$ nuclei fraction in different areas of the heart and at different developmental time points after birth, which is of particular relevance for studying cardiac regeneration. For our analysis, we have chosen an early postnatal time point at which the heart still has proliferative capacity (P3), a later postnatal time point at which the heart is just losing its potential to regenerate (P7) and adult age (9 weeks) when the heart is terminally differentiated. Figure 4a displays epifluorescence pictures of transgenic hearts at P0, P3, P7, and at adult stage [9 weeks $(9 \mathrm{~W})]$. While the expression level of H2B-mCh increased in the ventricle during postnatal development, it did not change in atria (Fig. 4a, close-ups).

For quantification of $\mathrm{CM}$ nuclei fractions, we analyzed heart sections for $\mathrm{H} 2 \mathrm{~B}-\mathrm{mCh}^{+}$nuclei and total cardiac nuclei. We assumed that due to the occurrence of binucleated $\mathrm{CMs}$, there would be a difference between quantification of $\mathrm{CM}$ nuclei based on either transverse or cross sections. In a cross section, only one of the two nuclei of a binucleated $\mathrm{CM}$ can be visible, while in longitudinal sections the
A
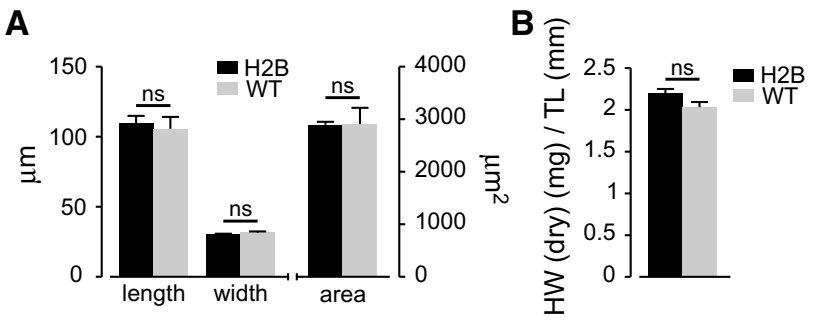

C

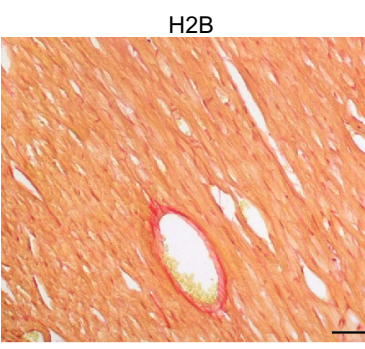

WT

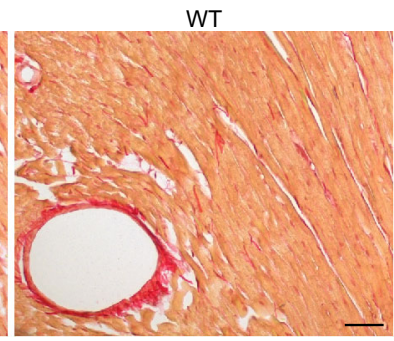

D

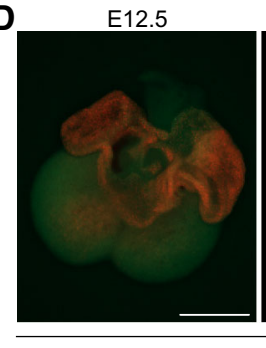
E15.5 E18.5
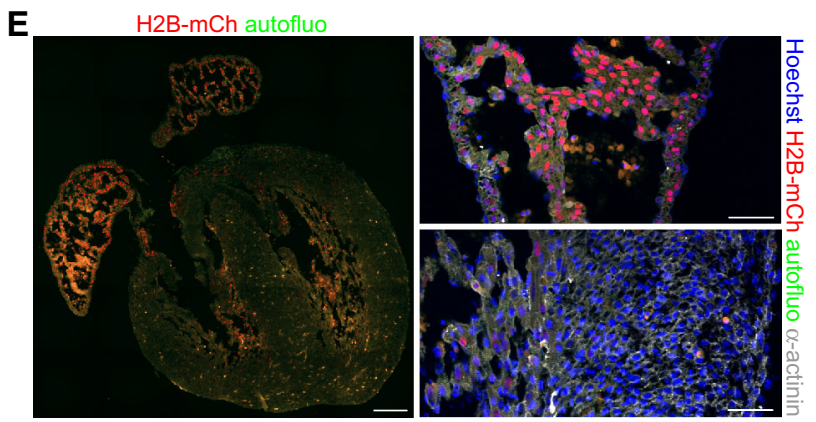

Fig. 3 Assessment of cardiac morphology in H2B-mCh mice and H2B-mCh expression during embryonic development. a Exclusion of hypertrophic effects of the transgene in Langendorff dissociated adult CMs. $N=3$ animals per group; $n>110$ CMs per group. b Comparison of heart weight to tibia length between 10-week-old male aMHC-H2B-mCh transgenic and wt littermates $(n=3)$. c Sections of $\mathrm{H} 2 \mathrm{~B}-\mathrm{mCh}$ and wt hearts stained with Picrosirius red for collagen. Scale bar $100 \mu \mathrm{m}$. d Macroscopic pictures of E12.5, E15.5, and E18.5 $\mathrm{H} 2 \mathrm{~B}-\mathrm{mCh}$ transgenic hearts illustrate the expression of the fusion protein in atrial and some ventricular CM nuclei. Scale bar $500 \mu \mathrm{m}$. e Section of an E15.5 H2B-mCh heart (left picture) displays strong expression in atrial $\mathrm{CM}$ nuclei (right upper picture) and weak expression in some ventricular trabecular CMs (right lower picture). Scale bars $200 \mu \mathrm{m}$ (overview), $50 \mu \mathrm{m}$ (close-ups)

chance to detect both nuclei is limited by section thickness. Therefore, both methods could be error-prone and either underestimate or overestimate the number of CMs. To determine the difference of counting transverse or cross sections on actual $\mathrm{CM}$ numbers, we first quantified the 
Table 1 Hemodynamics of H2B-mCh hearts

\begin{tabular}{lccl}
\hline Parameter & WT & H2B-mCh & Significance $(p)$ \\
\hline Heart rate $(\mathrm{bpm})$ & $471.8 \pm 42.6$ & $499.7 \pm 75.4$ & 0.61 \\
Maximum pressure $(\mathrm{mmHg})$ & $99.1 \pm 7.9$ & $99.6 \pm 3.3$ & 0.94 \\
End-diastolic pressure $(\mathrm{mmHg})$ & $5.5 \pm 5.9$ & $4.9 \pm 3.6$ & 0.88 \\
Stroke volume $(\mu \mathrm{L})$ & $25.5 \pm 15.1$ & $23.5 \pm 7.1$ & 0.85 \\
Ejection fraction $(\%)$ & $78.1 \pm 12.4$ & $69.4 \pm 9.0$ & 0.38 \\
Cardiac output $(\mu \mathrm{L} / \mathrm{min})$ & $11,787.5 \pm 6295.5$ & $11,413.9 \pm 1549.7$ & 0.93 \\
dPdt max $(\mathrm{mmHg} / \mathrm{s})$ & $9956.8 \pm 1036.3$ & $9425.9 \pm 1445.6$ & 0.63 \\
dPdt min $(\mathrm{mmHg} / \mathrm{s})$ & $-10,347.2 \pm 1853.1$ & $-10,052.9 \pm 1290.4$ & 0.83 \\
\hline
\end{tabular}

$d P d t$ max maximum rate of pressure change in the ventricle, $d P d t$ min minimal rate of pressure change in the ventricle, $m m H g$ millimeter of mercury (data are mean $\pm \mathrm{SEM}, n=3$ for all) fraction of $\mathrm{CM}$ nuclei in volume 3D-reconstitutions of Z-stacks of thick $(50 \mu \mathrm{m})$ slices from adult $\alpha \mathrm{MHC}-\mathrm{H} 2 \mathrm{~B}$ $\mathrm{mCh}$ hearts without taking into account the degree of binucleation. Nuclei were stained with TO-PRO3 iodide, cell borders were visualized by WGA staining and $\mathrm{CM}$ nuclei were identified by nuclear $\mathrm{H} 2 \mathrm{~B}$-mCh fluorescence (Fig. 4c). The effect of quantification of longitudinal or cross cardiac sections was determined by generating virtual sections from the 3D volume view (Fig. 4c). Interestingly, both methods of quantification gave very similar results compared to the fraction of CM nuclei obtained by analyzing the 3D reconstitution (Fig. 4d).

To assess potential regional differences in the fraction of CM nuclei at each time point, which could help identifying the most appropriate region(s) for proliferation inducing therapeutic approaches, different zones along the longitudinal axis of the heart (zone 1-3) were analyzed as depicted in Fig. 5a; in these areas we further distinguished between atria, left (LV) and right ventricle (RV) as well as between the compact and trabecular zone. The CM nuclei fractions were quantified by counting $\mathrm{H} 2 \mathrm{~B}-\mathrm{mCh}^{+}$nuclei and total number of nuclei. Quantification revealed a significant decrease in the fraction of CM nuclei from P3 to $9 \mathrm{~W}$ in all analyzed areas (Fig. 5b). At P3 a significantly higher fraction of CM nuclei was observed in atria compared to $\mathrm{LV}$, while the CM nuclei fractions did not differ significantly between the ventricles. At P7 the difference between $\mathrm{CM}$ nuclei fractions in atria and ventricles was evened and in $9 \mathrm{~W}$ old hearts the fraction of CM nuclei was $\sim 30 \%$ in atria as well as in both ventricles (Fig. 5b). These values were verified by flow cytometric analysis of isolated nuclei from hearts of 10-week-old transgenic animals, in which $25.0 \pm 4.2 \%$ of nuclei turned out to be H2B-mCh ${ }^{+}$(Fig. 2g). Regarding regional differences within the ventricles, a significantly smaller portion of $\mathrm{CM}$ nuclei was found in the trabecular zone compared to the compact zone on P3 and P7 (Fig. 5c). However, after compaction in adult hearts, the portion of $\mathrm{CM}$ nuclei was comparable in both zones (Fig. 5c). Along the apical- to basal heart axis, the $\mathrm{CM}$ nuclei fraction in the ventricles did not differ significantly (Fig. 5d). However, there was a slight tendency toward higher fractions of $\mathrm{CM}$ nuclei in apical direction. For accurate determination of CM number, the nuclearity of the CMs had to be taken into account, because most of the CMs in mice are binuclear [37].

\section{Determination of CM nuclearity and CM fractions at postnatal stages}

Shortly after birth CMs, undergo cell cycle variations resulting in binucleated and polyploid cells. These CMs are thought to be terminally differentiated [35], while there is evidence that mononucleated, diploid CMs have the potential to divide after stimulation of neuregulin/ErbB4signaling [7] or after experimental myocardial lesion [7, 33]. To determine the actual number of CMs from the fraction of CM nuclei and due to the potential relevance of mononuclear CMs for regenerative approaches in the heart, we quantified the fraction of bi- and mononucleated CMs at different time points and in different regions of the heart. However, the degree of binucleated CMs could not be determined accurately with either cross sections or longitudinal sections compared to 3D reconstitution (Fig. 6a). This is due to general heart geometry and the orientation of CMs in heart tissue, in which muscle fibers are curved and CMs are oriented at different angles. Furthermore, in an optimal standard cross section $(5-10 \mu \mathrm{m})$ binucleated cells cannot be detected, because usually only one nucleus is sectioned at a time. In longitudinal sections, the thickness of a standard section $(5-10 \mu \mathrm{m})$ limits the detection of both nuclei in binucleated CMs, if the CMs are not oriented in parallel to the sectioning plane or nuclei of one $\mathrm{CM}$ are not completely located in the same plane.

To accurately determine the ratio of mononucleated to bi- or multinucleated CMs, we followed two different approaches. The number of nuclei per $\mathrm{CM}$ was counted in enzymatically dissociated transgenic hearts at P3, P7, and in Langendorff-dissociated hearts at $9 \mathrm{~W}$. The 
A

H2B-mCh autofluo TL

웅

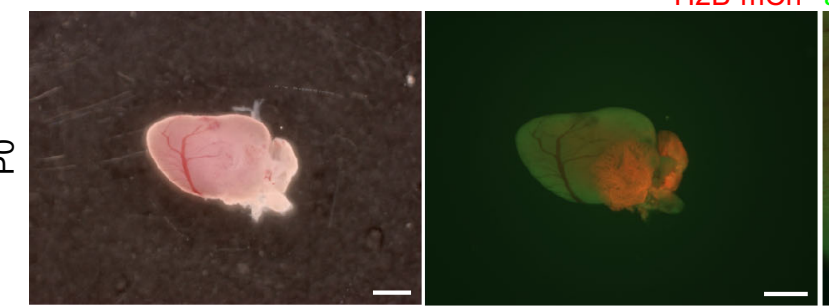

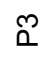

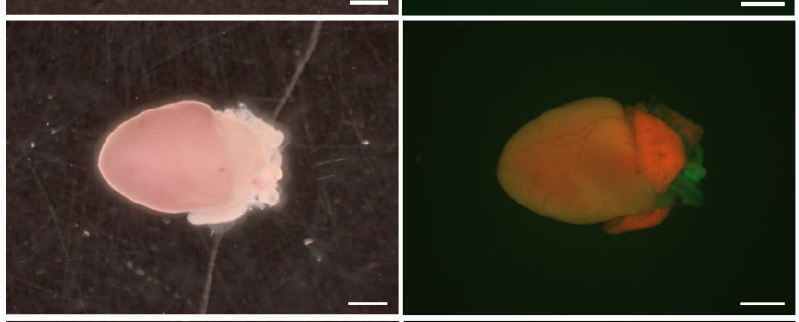

ล

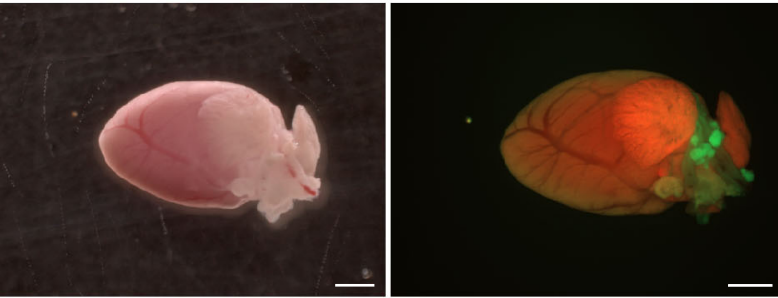

๘े
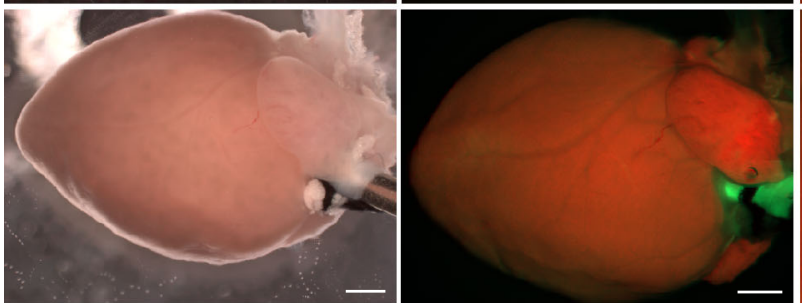

Hoechst H2B-mCh $\alpha$-actinin

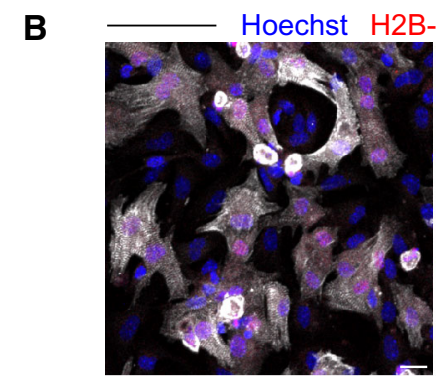

ventricular cells

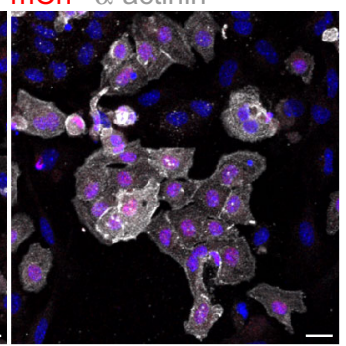

atrial cells

D

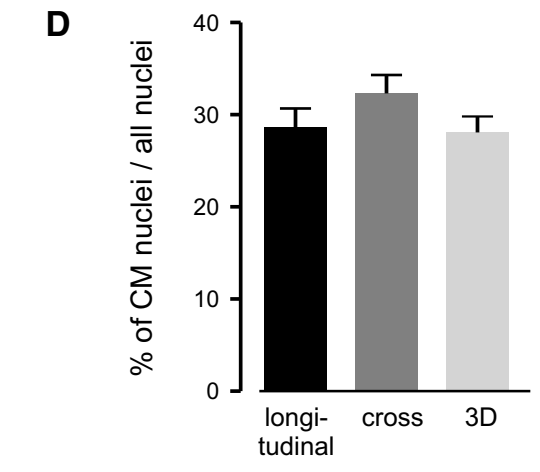

atrial cells
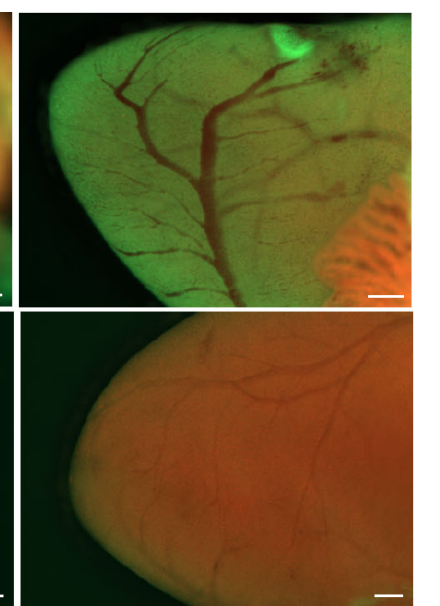

atrium

ventricle

\section{C}

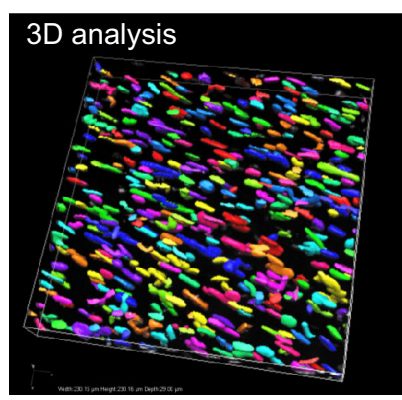

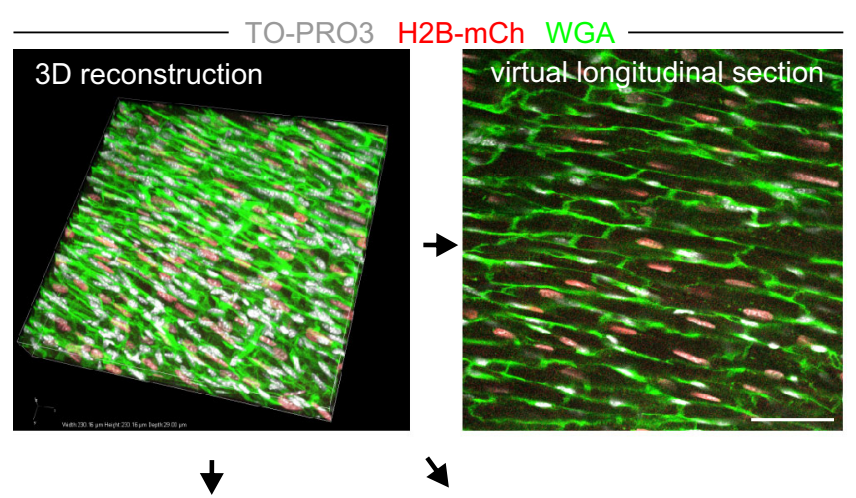

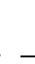

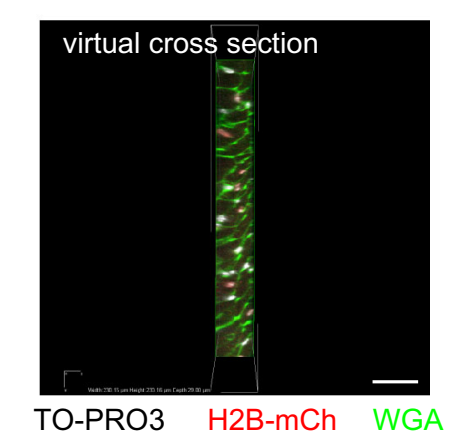


4Fig. $4 \mathrm{H} 2 \mathrm{~B}-\mathrm{mCh}$ transgene expression in postnatal mouse hearts. a Epifluorescence pictures of transgenic $\alpha \mathrm{MHC}-\mathrm{H} 2 \mathrm{~B}-\mathrm{mCh}$ hearts at different postnatal days. Scale bars $1 \mathrm{~mm}$ in the overview pictures, $200 \mu \mathrm{m}$ in close-ups of atrium and ventricle. b $\alpha$-Actinin staining (white) of dissociated ventricular (left) and atrial CMs (right) of aMHC-H2B-mCh transgenic hearts proves expression of the fusion protein at P3. Scale bars $20 \mu \mathrm{m}$. c Illustration of three methods to quantify the portion of $\mathrm{CM}$ nuclei in thick slices of the heart. The 3Danalysis of $\mathrm{H} 2 \mathrm{~B}-\mathrm{mCh}^{+}$nuclei in correlation to all ToPro- $3^{+}$nuclei reflects the real fraction of $\mathrm{CM}$ nuclei. Virtual longitudinal sections $(10 \mu \mathrm{m}$ distance, $3-4$ sections per slice) or virtual cross sections [40 $\mu \mathrm{m}$ distance, (4-5 sections per slice)] were analyzed. Scale bars $50 \mu \mathrm{m}$. Colored 3D image exemplarily depicts fragmentation of ToPro- $3^{+}$nuclei. d Quantification of the fraction of $\mathrm{CM}$ nuclei revealed similar results with all three methods $(n=3$, two z-stacks per heart)

quantification of isolated CMs revealed a strongly increased binucleation index in ventricular $\mathrm{CMs}$ from $11.6 \pm 1.6 \%$ at $\mathrm{P} 3$, to $65.5 \pm 1.7 \%$ at $\mathrm{P} 7$, and to $89.5 \pm 2.0 \%$ at $9 \mathrm{~W}$ (Fig. 6b). In atrial CMs, we determined only a slight increase in binuclearity from P3 $(14.2 \pm 2.2 \%)$ to $\mathrm{P} 7(32.0 \pm 1.2 \%)$. The fraction of multinucleated CMs exceeding binucleation was $<1 \%$ in all cases. In parallel, confocal images of thick slices $(50 \mu \mathrm{m})$ of transgenic hearts at $9 \mathrm{~W}$ were analyzed as Z-stacks to exclude a potential selective loss of mono- or binucleated CMs during the Langendorff isolation procedure. The slices were stained with WGA to visualize cell borders (Fig. 6c). Only CMs that lay completely within the z-stack (WGA staining visible in every dimension) were analyzed.

The quantifications of Z-stacks at adult stage allowed a separate analysis of the binucleation index in cells from the left $(77.2 \pm 4.4 \% ; n=5)$ and right ventricle $(77.8 \pm 3.4 \% ; n=5)$ and the atrium $(14.4 \pm 4.3 \%$; $n=4$ ) (Fig. 6b), thereby supporting the results obtained by the analysis of the Langendorff dissociated CMs. The fraction of mononucleated cells in the ventricles, as determined by this method, was significantly higher $(p=0.034)$ than with enzymatic dissociation.

By taking into account the portion of binucleated CMs, as assessed by Z-stack analysis, enzymatic cell separation and Langendorff dissociation, we were able to calculate the portions of CM cells in atria and ventricles at the selected time points (Fig. 6d). Due to the low degree of binucleation in atria, the fraction of $\mathrm{CMs}$ in this compartment was significantly higher at all analyzed stages (P3: $48.7 \pm 1.4 \%$; P7: $36.1 \pm 3.9 \%$; $9 \mathrm{~W}: 30.1 \pm 1.6 \%$ ) compared to ventricles at P3 $(39.2 \pm 2.6 \%)$, P7 $(25.8 \pm 0.1 \%), 9 \mathrm{~W}(16.1 \pm 0.2 \%$ dissociation $)$ and $\mathrm{LV}$ $(18.1 \pm 0.6 \%$ thick slices $)$ and $\mathrm{RV}(17.3 \pm 0.6 \%$ thick slices), respectively.

We also explored, whether H2B-mCh expression correlates to DNA content and could therefore be used as a
A

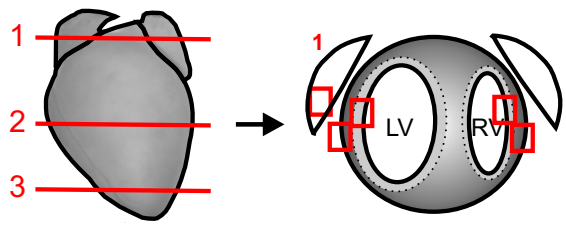

\section{C}

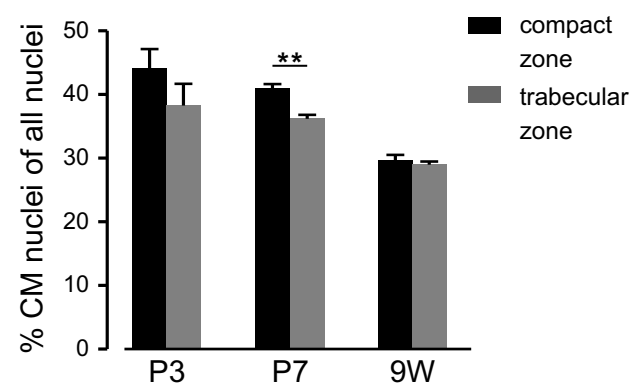

Fig. 5 Determination of the percentage of $\mathrm{CM}$ nuclei at different developmental stages. a Scheme illustrates the strategy used for analyzing the $\mathrm{CM}$ nuclei proportion in different regions of the heart. b Quantification of the percentage of $\mathrm{CM}$ nuclei at different
B
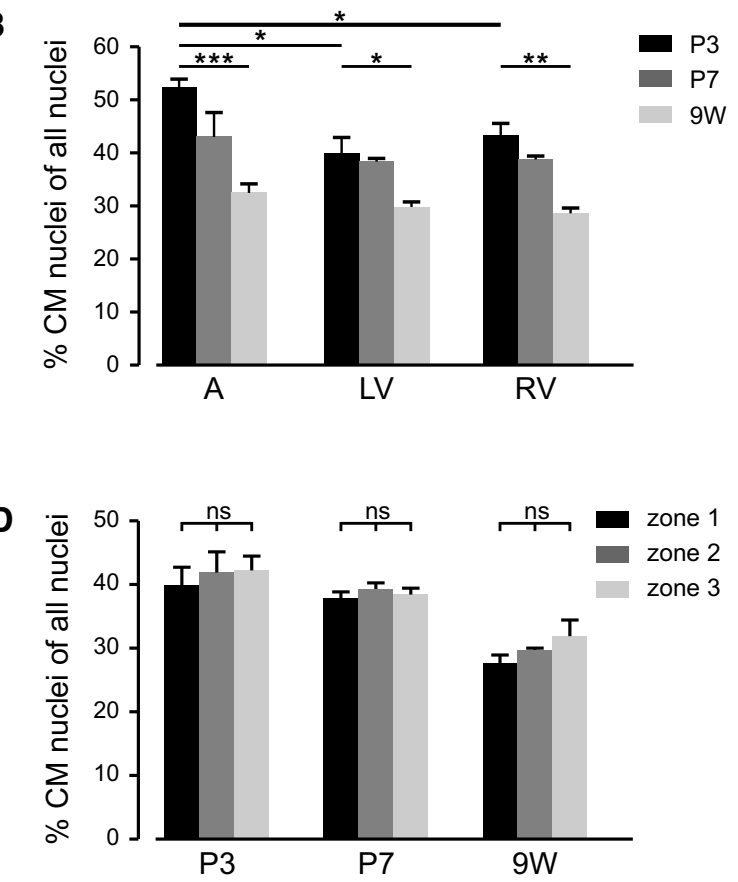

developmental stages. $A$ atrium, $L V$ left ventricle, $R V$ right ventricle, $(n=3)$. c Compact zone compared to trabecular region. $\mathbf{d}$ Fraction of CMs in different zones of the heart as described in (a) 
A

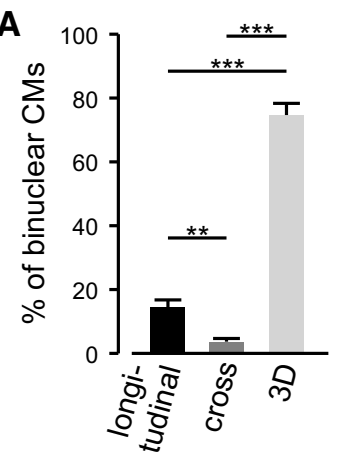

C
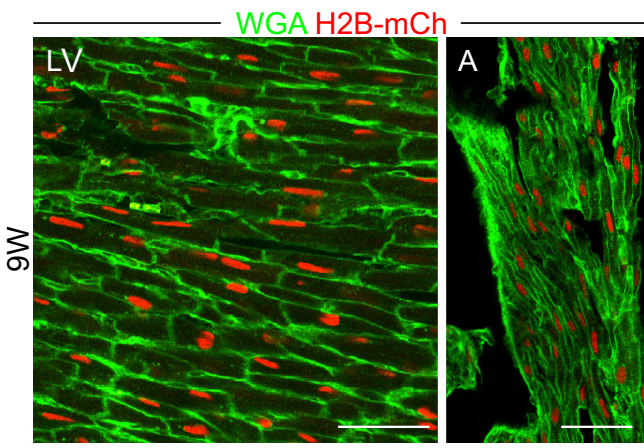
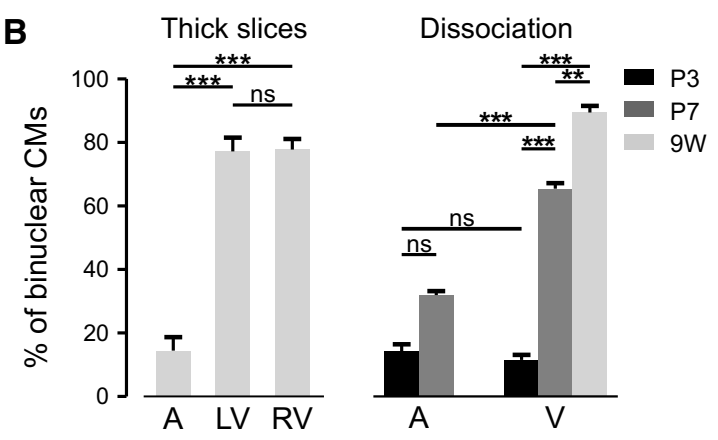

D
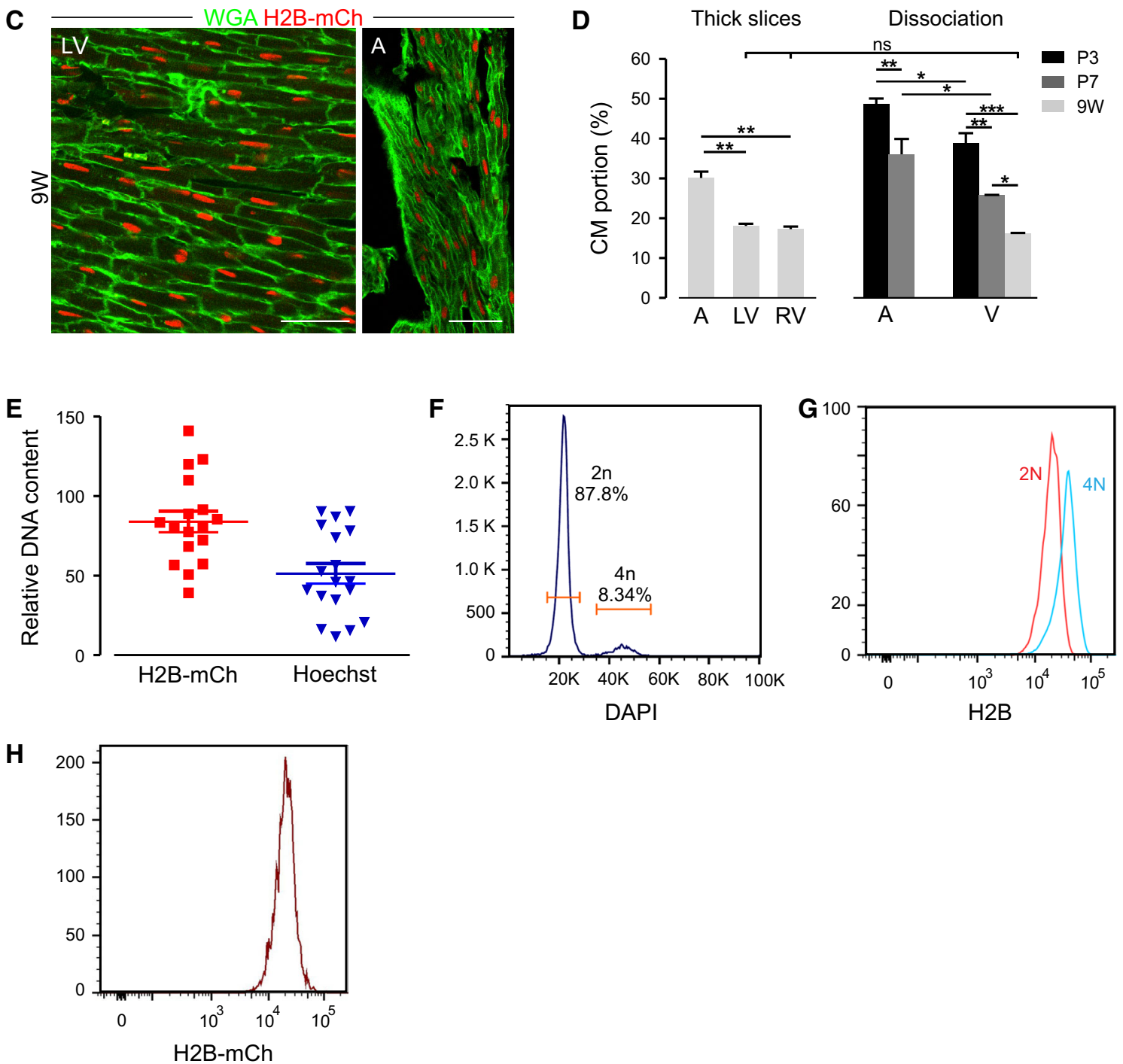

direct readout for endoreduplication. However, neither Hoechst staining of thick sections of the LV (Fig. 6e) nor isolation of CM nuclei followed by nuclear staining and flow cytometry (Fig. 6f-h) yielded a direct correlation between H2B-mCh signal intensity and DNA content.

\section{Easy identification of the border zone} after cryoinjury of the heart

After experimental myocardial infarction, it is challenging to clearly identify and quantify infarct size and surviving 
4 Fig. 6 Determination of nuclearity and DNA content in $\mathrm{H} 2 \mathrm{~B}-\mathrm{mCh}$ CMs. a Portion of CMs with two nuclei in one section $(n=3,2$ Z-stacks per heart) determined in thick slice 3D reconstruction and virtual longitudinal and cross sections. b Quantification of the percentage of binuclear CMs in $L V$ left ventricle and $R V$ right ventricle at P3 $(n \geq 3), \mathrm{P} 7(n \geq 3), 9 \mathrm{~W}(n=3)$, and in $A$ atrium at $9 \mathrm{~W}(n=2)$. Quantification was performed in z-stacks of thick slices for $9 \mathrm{~W}$ and cell dissociation for P3, P7, and $9 \mathrm{~W}$. c Single z-layers of thick heart slices show differences in the tissue morphology of the $L V$ left ventricle and $A$ atrium at adult stage. Membranes are stained with Wheat germ agglutinin (green). Scale bar $50 \mu \mathrm{m}$. d Calculation of the portion of CMs taking into account the numbers of Fig. 5b and the grade of binuclearity from thick slices and cell dissociation. e Relative DNA content of Langendorff isolated CMs as assessed by $\mathrm{H} 2 \mathrm{~B}-\mathrm{mCh}$ intensity or Hoechst intensity, respectively. f Representative flow cytometric analysis of the ploidy of $\mathrm{H} 2 \mathrm{~B}-\mathrm{mCh}$ single adult DAPI positive nuclei. $\mathrm{g}$ Representative flow cytometric analysis of the fluorescence intensity of $\mathrm{mCh}$ from diploid and tetraploid $\mathrm{CM}$ nuclei. $\mathbf{h}$ Representative flow cytometric analysis of the fluorescence intensity of $\mathrm{mCh}^{+}$nuclei

CMs in the border zone and infarcted area. This is especially true for islands of surviving CMs within the infarct. Additionally, staining for antigens in the infarct zone is difficult due to high background issues. We thought that these difficulties could be addressed by the prominent in vivo labeling of CM nuclei and therefore analyzed cardiac sections 10 days after the injury $(n=2)$. The lesioned area and the native myocardial tissue were easy to discriminate macroscopically by H2B-mCh signals (Fig. 7a). Staining of sections from lesioned transgenic hearts revealed a good demarcation of the infarct area and the viable myocardium with $\mathrm{H} 2 \mathrm{~B}-\mathrm{mCh}^{+}$nuclei (Fig. 7b). Specificity of the H2B-mCh signal was corroborated by staining for $\alpha$-actinin (Fig. 7c) and cardiac troponin $\mathrm{T}$ (Fig. 7d).

The expression of the fusion protein further facilitates the identification of nuclei from border zone CMs. Especially in cross sections, the reliable identification of CM nuclei is limited without the use of a specific nuclear marker as the CM nuclei are not always centered within the cells (Fig. 7e, arrows). Similarly, individual CMs or small islands of surviving $\mathrm{CMs}$, which are hard to detect within the lesioned area, are easily detectable by their H2B-mCh nuclear signal (Fig. 7f, arrows).

In summary, the aMHC-H2B-mCh mouse line facilitates the reliable identification of CM nuclei after myocardial infarction and eases the estimation of the lesion size.

\section{An in vitro assay for screening for proliferation- inducing substances in CMs}

A potentially very interesting strategy to regenerate lost heart muscle is based on re-induction of proliferation in pre-existing CMs. For this purpose preferentially neonatal stages are used, as at this stage some proliferative activity is still present in CMs and these cells appear to be more amenable to cell cycle-modulating interventions. To establish an in vitro and in vivo screening system for such factors and substances, we crossed the $\alpha \mathrm{MHC}-\mathrm{H} 2 \mathrm{~B}-\mathrm{mCh}$ mice with mice expressing the CAG-eGFP-anillin transgene [15], which visualizes cell cycle activity with high resolution of M-phase. In this double transgenic mouse line, CMs can be identified by nuclear expression of H2B$\mathrm{mCh}$ and their cell cycle status by localization of the eGFPanillin fusion protein (Fig. 8a). As proof of concept, we used postnatal CMs derived from double transgenic mice to test the influence of substances on cell cycle activity and proliferative behavior in vitro. We chose $\mathrm{P} 2$ mice for the experiment, as this age demarcates the time frame in which CMs are still proliferative, but will soon undergo the transition to binucleation, starting around $\mathrm{P} 4$. As the experiment lasts 3 days, which corresponds to P3-P5, both regular proliferation as well as cell cycle variations will take place, thereby providing a challenge for the detection system to correctly distinguish between the two states. Ventricles from P2 mice were dissociated and the cells were transfected with either microRNA 199, (miR-199) which was reported to strongly enhance the rate of proliferation in postnatal CMs [10] or scramble miRs as a negative control. By counting eGFP-anillin expressing $\mathrm{CMs}$, which could be identified according to their H2B$\mathrm{mCh}$ fluorescence, increased cell cycle activity after treatment with miR-199 compared to the scramble miR control could be directly detected without further stainings (Fig. 8b). While in transfections with scramble miRs, a basal cell cycle activity $(6.6 \%)$ could be determined, this was significantly $(p=0.0015)$ enhanced $(19.1 \%)$ after transfection with miR-199 (Fig. 8c). Importantly, the fraction of non-nuclear localizations of the eGFP-anillin signal, such as contractile rings and midbodies (Fig. 8d), which are indicative for cell division [15] and were verified by staining for Aurora B kinase (Fig. 8e), was increased after miR-199 treatment compared to the control (Fig. 8f). Further we noticed an increase in the fraction of binucleated CMs in miR-199-treated CMs (Fig. 8g). As the strength of our system is live-tracking of cell cycle progression, we performed video microscopy, which revealed both CMs' divisions (Suppl. Video 2) as well as binucleation (Suppl. Videos $3+4$ ) taking place. Binucleation was determined as $17.7 \%$ of total CMs for miR-199 treatment and only $9.8 \%$ for controls. This experiment demonstrates that the $\alpha \mathrm{MHC}-\mathrm{H} 2 \mathrm{~B}-\mathrm{mCh} / \mathrm{CAG}-\mathrm{eGFP}-a n i l l i n$ system not only enables the identification of proliferation, but also of cell cycle variations such as binucleation. Therefore, it is ideally suited for screening of cell cycle-modifying substances in CMs. 
Fig. 7 Visualization of $\mathrm{CM}$ nuclei after cardiac infarction. a Macroscopic pictures of an aMHC-H2B-mCh heart 10 days after cryoinjury. Close-up depicts border zone region. Scale bars overview: $1 \mathrm{~mm}$, close-up: $200 \mu \mathrm{m}$. b Section of a cryo-infarcted $\alpha$ MHC-H2B$\mathrm{mCh}$ heart. H2B-mCh signals are only abundant in intact $\mathrm{CMs}$ and are lost in the infarcted region. Scale bar $200 \mu \mathrm{m}$. (c, d) $\alpha \mathrm{MHC}-\mathrm{H} 2 \mathrm{~B}-\mathrm{mCh}^{+}$heart section stained with either $\alpha$ actinin (c) or cardiac Troponin $\mathrm{T}$ (d) to identify CMs. Good colocalization between the cardiac markers and $\mathrm{H} 2 \mathrm{~B}-\mathrm{mCh}$ in the border zone. Scale bars $20 \mu \mathrm{m}$. e H2B-mCh expression

facilitates the identification of $\mathrm{CM}$ nuclei in cross sections of the border zone. Arrows depict $\mathrm{CM}$ nuclei, which are not centered in the cell. Scale bar $20 \mu \mathrm{m}$. f H2B-mCh expression facilitates the identification of individual surviving $\mathrm{CMs}$ (arrows) within the lesioned area. Scale bar $20 \mu \mathrm{m}$
A
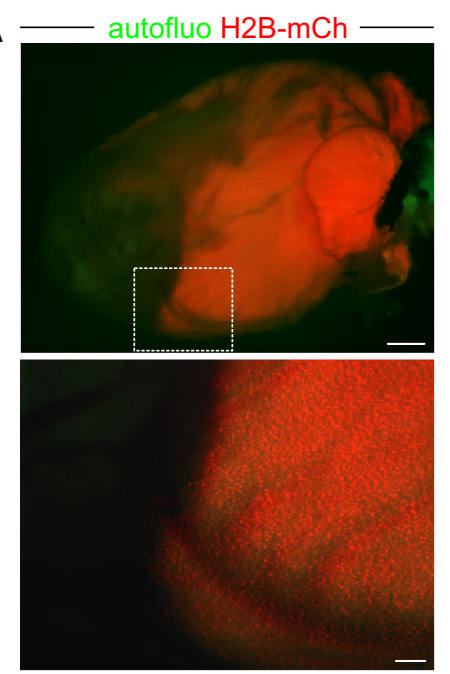

B
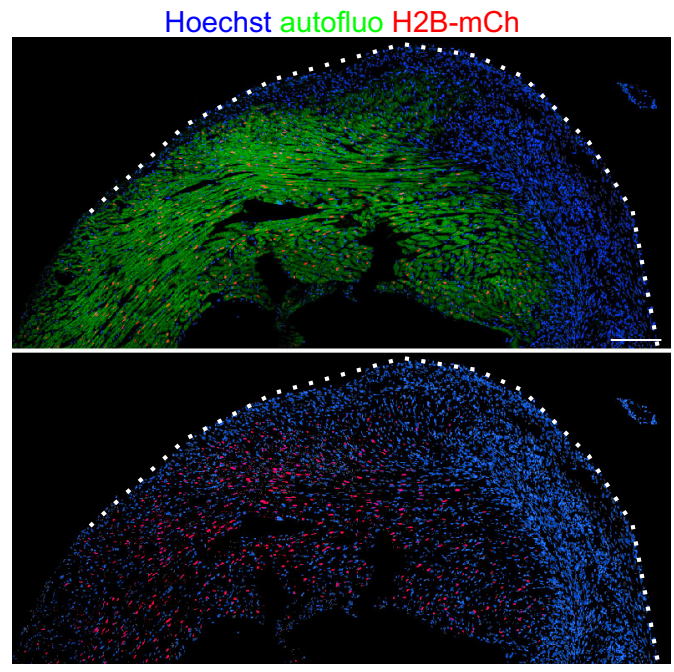

C

Hoechst H2B-mCh $\alpha$-actinin

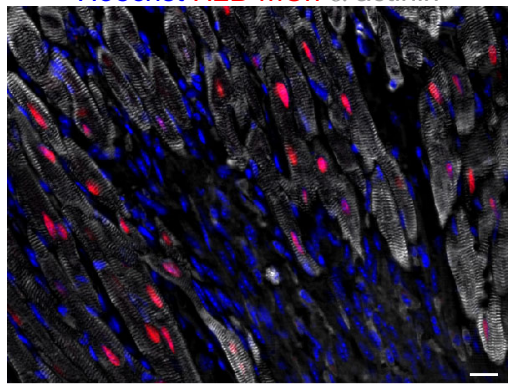

D

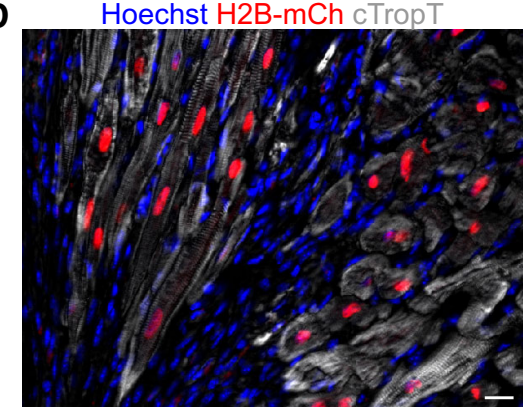

E
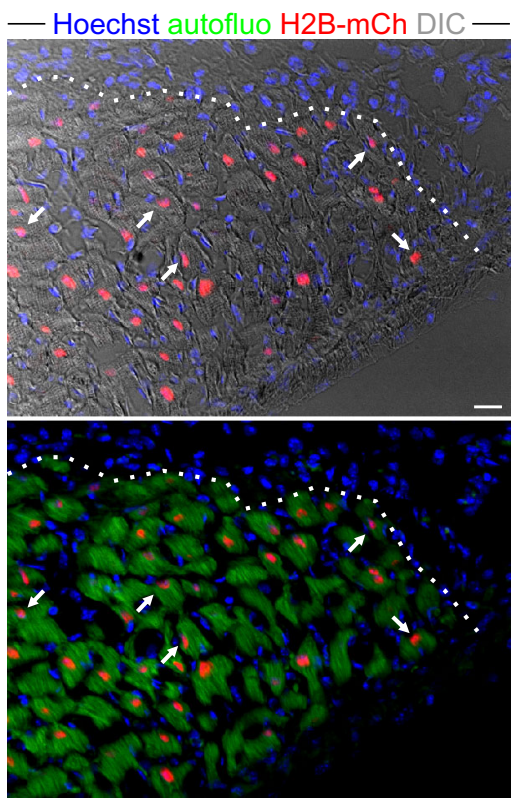

\section{F - Hoechst autofluo H2B-mCh DIC -}
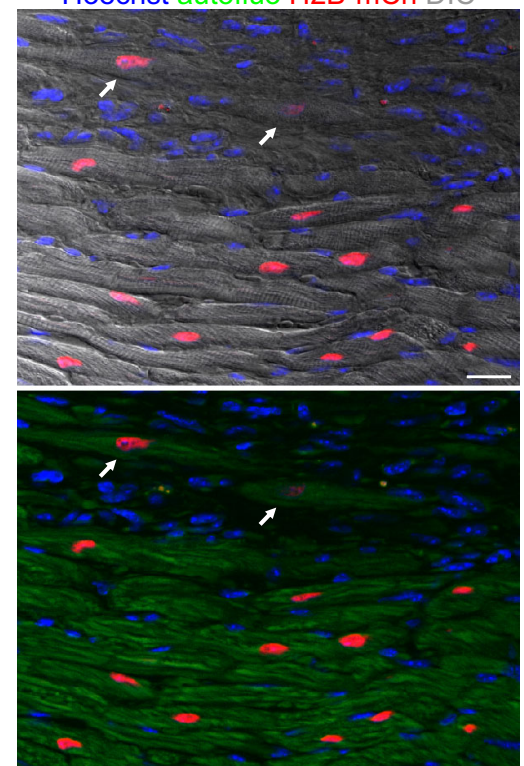

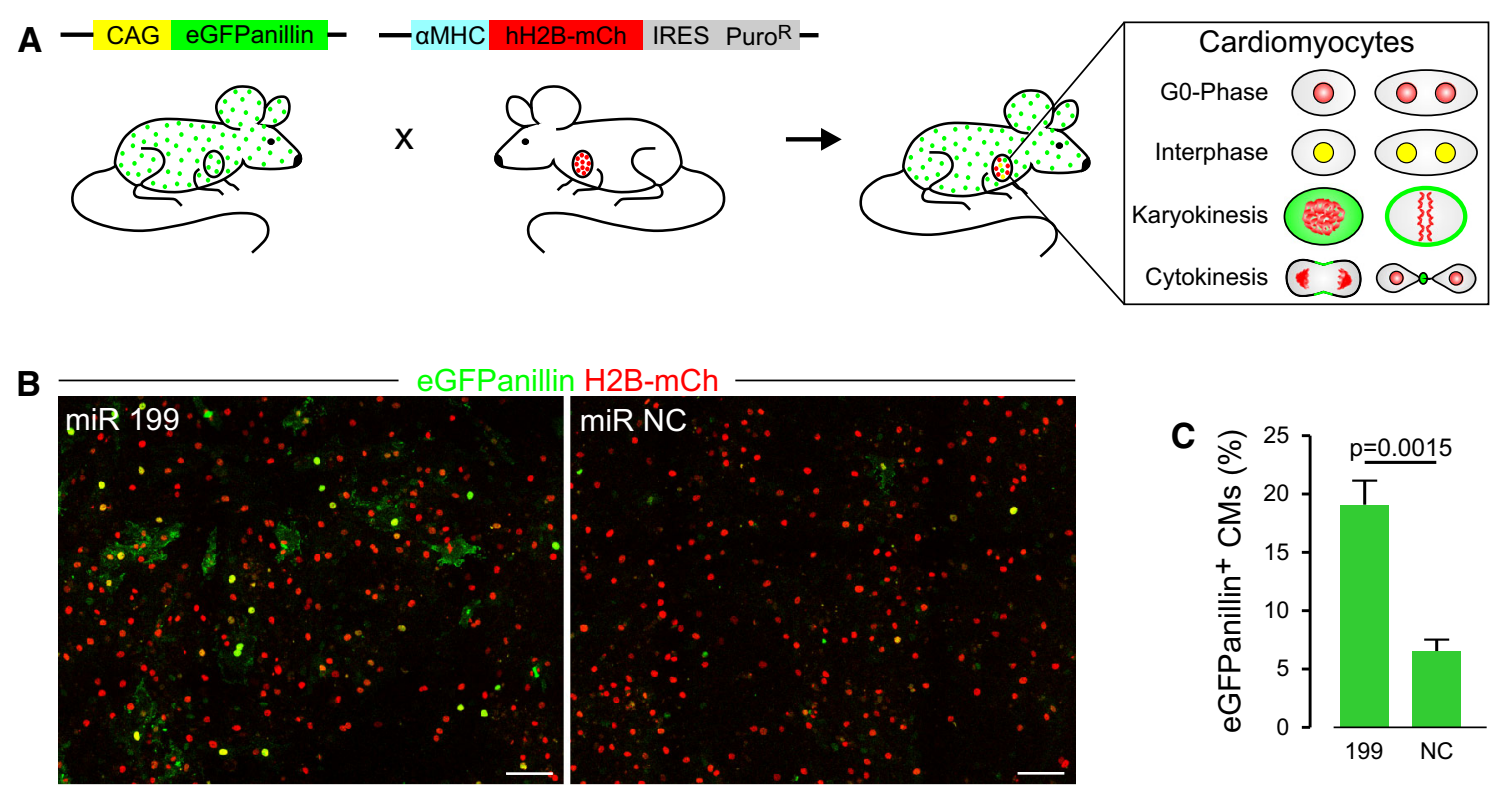
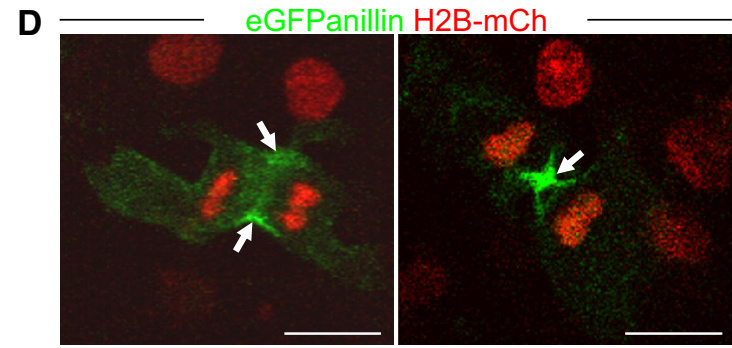

$\mathbf{F}$

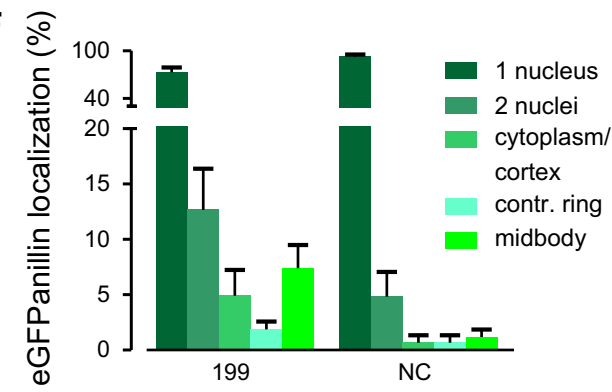

Fig. 8 Assay for screening the effects of cell cycle modifying substances on CMs. a Scheme depicts the cross-breeding of aMHC$\mathrm{H} 2 \mathrm{~B}-\mathrm{mCh}$ mice with the CAG-eGFP-anillin proliferation indicator mouse. Depending on the cell cycle status, $\mathrm{CMs}\left(\mathrm{H} 2 \mathrm{~B}-\mathrm{mCh}^{+}\right.$nuclei) express eGFP-anillin in different subcellular localizations. b Fluorescence pictures of dissociated $\alpha \mathrm{MHC}-\mathrm{H} 2 \mathrm{~B}-\mathrm{mCh} / \mathrm{CAG}-\mathrm{eGFP}-$ anillin double transgenic hearts $(\mathrm{P} 2)$, transfected with the cell cyclemodifying miR-199 and a miR-NC. Scale bars $100 \mu \mathrm{m}$. c Quantification of eGFP-anillin expression in miR-treated CMs $72 \mathrm{~h}$ after

\section{Discussion}

We have generated a novel transgenic model for the direct visualization of CM nuclei in vitro and in vivo. This system is based on the expression of a fusion protein consisting of
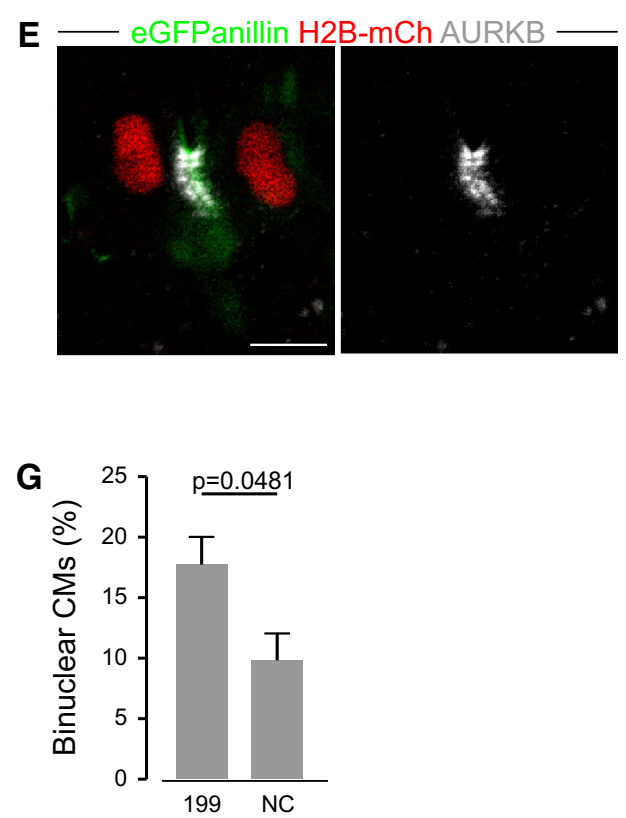

transfection ( $n \geq 3$ ). d Examples of CMs with cytokinesis-indicating eGFP-anillin localizations (arrows). Scale bars $100 \mu \mathrm{m}$. e Staining of eGFP-anillin/H2B-mCh CMs with the proliferation marker AURKB Aurora B kinase. Note the overlap between the M-phase specific localization of eGFP-anillin (green) contractile ring and Aurora B kinase (white). Scale bar $10 \mu \mathrm{m}$. f Analysis of different eGFP-anillin localizations in CMs after miR-treatment $(n \geq 3)$. g Portion of binuclear CMs $72 \mathrm{~h}$ after miR-transfection $(n \geq 3)$

the human histone $2 \mathrm{~B}$ and the red fluorescent protein $\mathrm{mCh}$ under transcriptional control of the CM specific Myh6 promoter, resulting in a nuclear signal. We showed the specific labeling of CM nuclei throughout our in vitro and in vivo experiments. 
As the mammalian heart consists of mono- and binucleated CMs, it was of great interest to determine the fraction and nuclearity of CMs in different regions of the mouse heart. The contribution of CMs to total number of heart cells in adult humans and rodents has been estimated to be $15-20 \%$ [37, 42]. However, for mice, even substantially higher estimates have been reported, assigning an exceptional role for this species [2]. We can clearly exclude this and confirm earlier estimates of $<15 \% \mathrm{CMs}$, which correspond to $<30 \% \mathrm{CM}$ nuclei [37, 43]. While previous quantifications were based on cell suspensions of dissociated hearts, our data are based on (1) flow cytometry of isolated nuclei, (2) quantification on tissue sections, (3) Langendorff isolation, as well as (4) 3D-reconstruction and (5) Z-stack analysis of cardiac slices. Cell isolation procedures in general cannot guarantee uniform extraction of all cell types. Cells that are more sensitive to enzymatic treatment are prone to be damaged, while cells surrounded by connective tissue are less likely to be dissolved from the tissue. By using Z-stacks of cardiac sections for quantitation, we found a significantly lower fraction of binuclear CMs as with enzymatic dissociation. A recent study in which histological sections were used also reported higher numbers of mononuclear CMs (24\% [33]) that match our observations of $\sim 25 \%$. In atria of adult mice, we found an even higher fraction of mononucleated cells of $\sim 86 \%$. The functional difference between mono- and binucleated CMs is unknown. However, there is evidence that mononucleated CMs are prone to enter the cell cycle after stimulation with neuregulin [7] or after cardiac lesion [33]. Interestingly, atrial mononuclear CMs display cell cycle activity after experimental lesion in the left ventricle in rats, which was attributed to either cell division [32] or increased binucleation [28].

Additionally, we also addressed the issue of CM fractions in the context of cardiac growth and spatial distribution in the heart, which is of relevance for studying cardiac regeneration as it has been shown that neonate mammalian hearts have a remarkable regenerative capacity [30]. We found a significantly higher proportion of CMs at P3 compared to P7 and adult mice which is most likely due to the extensive proliferation of fibroblasts taking place from P1 to P10 [37]. The spatial distribution of CMs revealed only a difference in atria of P3, P7, and adult mice, in which the fraction of CMs was significantly higher compared to the ventricles. This is due to the significantly lower fraction of binucleated cells in atria of mouse, which was $\sim 14 \%$ in adults.

A promising strategy for regeneration of lost CMs is the re-induction of CM division by application of proliferationinducing substances. However to identify such factors and to verify them in vivo, CM nuclei and cell division need to be unequivocally identified.
The advantages of our CM proliferation assessment assay, which is based on $\mathrm{H} 2 \mathrm{~B}-\mathrm{mCh} / \mathrm{eGFP}$-anillin compound transgenic mice, are the immediate $\mathrm{CM}$ nuclei identification and quantification of cell cycle activity due to the fluorescent labels and in particular the possibility to livemonitor cell cycle progression of CMs. In contrast, most commonly used assays use BrdU and stainings with combinations of Ki-67, pHH3, PCNA, and AuroraB-kinase [7, $9,10,41]$ which require fixation and prevent the direct follow-up of cell fate. As a proof of concept, we were able to verify the proliferation-inducing effect of miR-199, as previously published [10]. Due to the high resolution of M-phase, the eGFP-anillin assay also allows us to discriminate between cell division and cell cycle variations such as endoreduplication and acytokinetic mitosis leading to binucleation [15]. Surprisingly, treatment with miR-199 led to an increase of binucleated CMs, indicating induction of cell cycle variations. In summary, the H2B-mCh/eGFPanillin assay will provide crucial information about the true nature of cell cycle activity in the future.

Currently, identification of CMs is mostly based on staining of the contractile apparatus (cTropI, cTropT or $\alpha$ actinin) or on the use of transgenic mouse models such as aMHC-mCh mice [19] or $\alpha \mathrm{MHC}-\mathrm{Cre}$ inducible mice [34] which provide CM-specific cytoplasmic expression of fluorescence reporters [18]. As a caveat, these techniques do not allow a direct correlation of nuclei to the marked CMs. False positive identification of a non-CM nucleus as cell cycle active CM nucleus may strongly alter a conclusion concerning the regeneration potential of adult CMs as the numbers of CMs with cell cycle activity are described to be very low (1 in 180,000) [36]. Indeed, a lack of proper identification of CM nuclei seems to be one of the major reasons for the high divergences in literature concerning the annual turnover rate of adult mouse $\mathrm{CMs}$, ranging from $0.76 \%$ [33] up to $80 \%$ [17].

Currently only one further transgenic mouse model, MHC-nLAC, has been described which enables a specific identification of $\mathrm{CM}$ nuclei by staining for $\beta$-galactosidase activity [38]. As a shortcoming, the nuclear signal is replaced by a diffuse cytoplasmic signal in CMs as soon as the nuclear envelope dissolves. This complicates quantifications of CM nuclei if cells enter M-Phase. The H2B-mCh mice described here have two advantages over the existing mouse lines, which use NLS or cytoplasmic expression of a CM specific marker: first, compared to lacZ as a marker [38] no additional staining step is needed, which is particulary important for live-imaging applications and second, due to the fusion to histone $\mathrm{H} 2 \mathrm{~B}$, $\mathrm{mCh}$ stays connected to the chromosomes during all phases of the cell cycle, a prerequisite for automatic cell tracking in a screening assay [27]. Furthermore, our mouse model can also be used to directly and specifically isolate CM nuclei 
for the analysis of epigenetic chromatin modifications, an approach recently established in CMs [13]. Taken together, our transgenic system provides a new technology for the identification of CM nuclei without any further treatment and for the isolation of CM nuclei for downstream applications. In combination with the eGFP-anillin system, it is a novel tool to specifically resolve controversial questions in the cardiac field, such as the ongoing debate, if adult CMs have the potential to divide. This mouse line will be useful for assessing proliferation in models of cardiac regeneration, for the differentiation between atrial and ventricular CMs during embryonic development, identification of CMs in screening assays for proliferation-inducing substances, transplantation experiments, and for the analysis of plasticity of adult CMs in future studies.

Acknowledgments We thank A. Nagy (Toronto, Canada) and M. Gertsenstein (Toronto, Canada) for providing the G4 mouse ESC line. We also would like to acknowledge S. Grünberg (Bonn, Germany), P. Freitag (Bonn, Germany), and J. Schmidt (Bonn, Germany) for technical assistance. This work was supported by the Deutsche Forschungsgemeinschaft [FOR 1352, FL276/6-1 to B.K.F., RO2279/ 2-2 to W.R] and the NRW International Graduate School BIOTECHPHARMA [fellowship to A.R.].

\section{Conflict of interest None declared.}

Open Access This article is distributed under the terms of the Creative Commons Attribution 4.0 International License (http:// creativecommons.org/licenses/by/4.0/), which permits unrestricted use, distribution, and reproduction in any medium, provided you give appropriate credit to the original author(s) and the source, provide a link to the Creative Commons license, and indicate if changes were made.

\section{References}

1. Ang KL, Shenje LT, Reuter S, Soonpaa MH, Rubart M, Field LJ, Galinanes M (2010) Limitations of conventional approaches to identify myocyte nuclei in histologic sections of the heart. Am J Physiol Cell Physiol 298:C1603-C1609. doi:10.1152/ajpcell. 00435.2009

2. Banerjee I, Fuseler JW, Price RL, Borg TK, Baudino TA (2007) Determination of cell types and numbers during cardiac development in the neonatal and adult rat and mouse. Am J Physiol Heart Circ Physiol 293:H1883-H1891. doi:10.1152/ajpheart.00514.2007

3. Baudino TA, Carver W, Giles W, Borg TK (2006) Cardiac fibroblasts: friend or foe? Am J Physiol Heart Circ Physiol 291:H1015-H1026. doi:10.1152/ajpheart.00023.2006

4. Bergmann O, Bhardwaj RD, Bernard S, Zdunek S, BarnabeHeider F, Walsh S, Zupicich J, Alkass K, Buchholz BA, Druid H, Jovinge S, Frisen J (2009) Evidence for cardiomyocyte renewal in humans. Science 324:98-102. doi:10.1126/science.1164680

5. Bergmann O, Jovinge S (2012) Isolation of cardiomyocyte nuclei from post-mortem tissue. J Vis Exp 65:e4205. doi:10.3791/4205

6. Bergmann O, Zdunek S, Alkass K, Druid H, Bernard S, Frisen J (2011) Identification of cardiomyocyte nuclei and assessment of ploidy for the analysis of cell turnover. Exp Cell Res 317:188-194. doi:10.1016/j.yexcr.2010.08.017
7. Bersell K, Arab S, Haring B, Kuhn B (2009) Neuregulin1/ErbB4 signaling induces cardiomyocyte proliferation and repair of heart injury. Cell 138:257-270. doi:10.1016/j.cell.2009.04.060

8. Brodsky VY, Chernyaev AL, Vasilyeva IA (1991) Variability of the cardiomyocyte ploidy in normal human hearts. Virchows Arch B Cell Pathol Incl Mol Pathol 61:289-294. doi:10.1007/ BF02890430

9. Engel FB, Hsieh PC, Lee RT, Keating MT (2006) FGF1/p38 MAP kinase inhibitor therapy induces cardiomyocyte mitosis, reduces scarring, and rescues function after myocardial infarction. Proc Natl Acad Sci USA 103:15546-15551. doi:10.1073/ pnas.0607382103

10. Eulalio A, Mano M, Dal FM, Zentilin L, Sinagra G, Zacchigna S, Giacca M (2012) Functional screening identifies miRNAs inducing cardiac regeneration. Nature 492:376-381. doi:10.1038/ nature 11739

11. Georgakopoulos D, Kass DA (2000) Estimation of parallel conductance by dual-frequency conductance catheter in mice. Am J Physiol Heart Circ Physiol 279:H443-H450

12. George SH, Gertsenstein M, Vintersten K, Korets-Smith E, Murphy J, Stevens ME, Haigh JJ, Nagy A (2007) Developmental and adult phenotyping directly from mutant embryonic stem cells. Proc Natl Acad Sci USA 104:4455-4460. doi:10.1073/pnas. 0609277104

13. Gilsbach R, Preissl S, Gruning BA, Schnick T, Burger L, Benes V, Wurch A, Bonisch U, Gunther S, Backofen R, Fleischmann BK, Schubeler D, Hein L (2014) Dynamic DNA methylation orchestrates cardiomyocyte development, maturation and disease. Nat Commun 5:5288. doi:10.1038/ncomms6288

14. Herget GW, Neuburger M, Plagwitz R, Adler CP (1997) DNA content, ploidy level and number of nuclei in the human heart after myocardial infarction. Cardiovasc Res 36:45-51. doi:10. 1016/S0008-6363(97)00140-5

15. Hesse M, Raulf A, Pilz GA, Haberlandt C, Klein AM, Jabs R, Zaehres H, Fugemann CJ, Zimmermann K, Trebicka J, Welz A, Pfeifer A, Roll W, Kotlikoff MI, Steinhauser C, Gotz M, Scholer HR, Fleischmann BK (2012) Direct visualization of cell division using high-resolution imaging of M-phase of the cell cycle. Nat Commun 3:1076. doi:10.1038/ncomms2089

16. Heusch G, Libby P, Gersh B, Yellon D, Bohm M, Lopaschuk G, Opie L (2014) Cardiovascular remodelling in coronary artery disease and heart failure. Lancet 383:1933-1943. doi:10.1016/ S0140-6736(14)60107-0

17. Hosoda T, D'Amario D, Cabral-Da-Silva MC, Zheng H, PadinIruegas ME, Ogorek B, Ferreira-Martins J, Yasuzawa-Amano S, Amano K, Ide-Iwata N, Cheng W, Rota M, Urbanek K, Kajstura J, Anversa P, Leri A (2009) Clonality of mouse and human cardiomyogenesis in vivo. Proc Natl Acad Sci USA 106:17169-17174. doi:10.1073/pnas.0903089106

18. Hsieh PC, Segers VF, Davis ME, MacGillivray C, Gannon J, Molkentin JD, Robbins J, Lee RT (2007) Evidence from a genetic fate-mapping study that stem cells refresh adult mammalian cardiomyocytes after injury. Nat Med 13:970-974. doi:10.1038/ $\mathrm{nm} 1618$

19. Jesty SA, Steffey MA, Lee FK, Breitbach M, Hesse M, Reining S, Lee JC, Doran RM, Nikitin AY, Fleischmann BK, Kotlikoff MI (2012) c-kit+ precursors support postinfarction myogenesis in the neonatal, but not adult, heart. Proc Natl Acad Sci USA 109:13380-13385. doi:10.1073/pnas.1208114109

20. Jugdutt BI (2003) Ventricular remodeling after infarction and the extracellular collagen matrix: when is enough enough? Circulation 108:1395-1403. doi:10.1161/01.CIR.0000085658.98621.49

21. Li F, Wang X, Capasso JM, Gerdes AM (1996) Rapid transition of cardiac myocytes from hyperplasia to hypertrophy during postnatal development. J Mol Cell Cardiol 28:1737-1746. doi:10. 1006/jmcc. 1996.0163 
22. Lyons GE, Schiaffino S, Sassoon D, Barton P, Buckingham M (1990) Developmental regulation of myosin gene expression in mouse cardiac muscle. J Cell Biol 111:2427-2436. doi:10.1083/ jcb.111.6.2427

23. Meckert PC, Rivello HG, Vigliano C, Gonzalez P, Favaloro R, Laguens R (2005) Endomitosis and polyploidization of myocardial cells in the periphery of human acute myocardial infarction. Cardiovasc Res 67:116-123. doi:10.1016/j.cardiores.2005.02.017

24. Mollova M, Bersell K, Walsh S, Savla J, Das LT, Park SY, Silberstein LE, Dos Remedios CG, Graham D, Colan S, Kuhn B (2013) Cardiomyocyte proliferation contributes to heart growth in young humans. Proc Natl Acad Sci USA 110:1446-1451. doi:10. 1073/pnas. 1214608110

25. Nag AC (1980) Study of non-muscle cells of the adult mammalian heart: a fine structural analysis and distribution. Cytobios 28:41-61

26. Nagy A, Rossant J, Nagy R, Bramow-Newerly W, Roder JC (1993) Derivation of completely cell culture-derived mice from early-passage embryonic stem cells. Proc Natl Acad Sci USA 90:8424-8428. doi:10.1073/pnas.90.18.8424

27. Nowotschin S, Eakin GS, Hadjantonakis AK (2009) Live-imaging fluorescent proteins in mouse embryos: multi-dimensional, multi-spectral perspectives. Trends Biotechnol 27:266-276. doi:10.1016/j.tibtech.2009.02.006

28. Oberpriller JO, Oberpriller JC, Aafedt BC (1987) Changes in binucleation and cellular dimensions of rat left atrial myocytes after induced left ventricular infarction. Am J Anat 179:285-290. doi:10.1002/aja.1001790310

29. Olivetti G, Cigola E, Maestri R, Corradi D, Lagrasta C, Gambert SR, Anversa P (1996) Aging, cardiac hypertrophy and ischemic cardiomyopathy do not affect the proportion of mononucleated and multinucleated myocytes in the human heart. J Mol Cell Cardiol 28:1463-1477. doi:10.1006/jmcc.1996.0137

30. Porrello ER, Mahmoud AI, Simpson E, Hill JA, Richardson JA, Olson EN, Sadek HA (2011) Transient regenerative potential of the neonatal mouse heart. Science 331:1078-1080. doi:10.1126/ science. 1200708

31. Roell W, Fan Y, Xia Y, Stoecker E, Sasse P, Kolossov E, Bloch W, Metzner H, Schmitz C, Addicks K, Hescheler J, Welz A, Fleischmann BK (2002) Cellular cardiomyoplasty in a transgenic mouse model. Transplantation 73:462-465. doi:10.1097/ 00007890-200202150-00022

32. Rumyantsev PP (1974) Ultrastructural reorganization, DNA synthesis and mitotic division of myocytes in atria of rats with left ventricle infarction. An electron microscopic and autoradiographic study. Virchows Arch B Cell Pathol 15:357-378

33. Senyo SE, Steinhauser ML, Pizzimenti CL, Yang VK, Cai L, Wang M, Wu TD, Guerquin-Kern JL, Lechene CP, Lee RT
(2013) Mammalian heart renewal by pre-existing cardiomyocytes. Nature 493:433-436. doi:10.1038/nature11682

34. Sohal DS, Nghiem M, Crackower MA, Witt SA, Kimball TR, Tymitz KM, Penninger JM, Molkentin JD (2001) Temporally regulated and tissue-specific gene manipulations in the adult and embryonic heart using a tamoxifen-inducible Cre protein. Circ Res 89:20-25. doi:10.1161/hh1301.092687

35. Soonpaa MH, Field LJ (1997) Assessment of cardiomyocyte DNA synthesis in normal and injured adult mouse hearts. Am J Physiol 272:H220-H226

36. Soonpaa MH, Field LJ (1998) Survey of studies examining mammalian cardiomyocyte DNA synthesis. Circ Res 83:15-26. doi:10.1161/01.RES.83.1.15

37. Soonpaa MH, Kim KK, Pajak L, Franklin M, Field LJ (1996) Cardiomyocyte DNA synthesis and binucleation during murine development. Am J Physiol 271:H2183-H2189

38. Soonpaa MH, Koh GY, Klug MG, Field LJ (1994) Formation of nascent intercalated disks between grafted fetal cardiomyocytes and host myocardium. Science 264:98-101. doi:10.1126/science. 8140423

39. Stegemann M, Meyer R, Haas HG, Robert-Nicoud M (1990) The cell surface of isolated cardiac myocytes-a light microscope study with use of fluorochrome-coupled lectins. J Mol Cell Cardiol 22:787-803

40. Tumbar T, Guasch G, Greco V, Blanpain C, Lowry WE, Rendl M, Fuchs E (2004) Defining the epithelial stem cell niche in skin. Science 303:359-363. doi:10.1126/science.1092436

41. Uosaki H, Magadum A, Seo K, Fukushima H, Takeuchi A, Nakagawa Y, Moyes KW, Narazaki G, Kuwahara K, Laflamme M, Matsuoka S, Nakatsuji N, Nakao K, Kwon C, Kass DA, Engel FB, Yamashita JK (2013) Identification of chemicals inducing cardiomyocyte proliferation in developmental stage-specific manner with pluripotent stem cells. Circ Cardiovasc Genet 6:624-633. doi:10.1161/CIRCGENETICS.113.000330

42. Vliegen HW, van der LA, Cornelisse CJ, Eulderink F (1991) Myocardial changes in pressure overload-induced left ventricular hypertrophy. A study on tissue composition, polyploidization and multinucleation. Eur Heart J 12:488-494

43. Walsh S, Ponten A, Fleischmann BK, Jovinge S (2010) Cardiomyocyte cell cycle control and growth estimation in vivo-an analysis based on cardiomyocyte nuclei. Cardiovasc Res 86:365-373. doi:10.1093/cvr/cvq005

44. Zaruba MM, Soonpaa M, Reuter S, Field LJ (2010) Cardiomyogenic potential of C-kit(+)-expressing cells derived from neonatal and adult mouse hearts. Circulation 121:1992-2000. doi:10.1161/CIRCULATIONAHA.109.909093 